\title{
A faint type of supernova from a white dwarf with a helium-rich companion
}

\author{
H. B. Perets, \\ Department of Particle Physics and Astrophysics, Faculty of Physics, The Weizmann Institute of Science, Rehovot 76100, \\ Israel; \\ and CfA Fellow, Harvard-Smithsonian Center for Astrophysics, 60 Garden Street, Cambridge, MA 02138, USA
}

A. Gal-Yam,

Department of Particle Physics and Astrophysics, Faculty of Physics, The Weizmann Institute of Science, Rehovot 76100, Israel

P. A. Mazzali,

Max-Planck Institut für Astrophysik, Karl-Schwarzschild-Str. 1, 85748 Garching, Germany;

Scuola Normale Superiore, Piazza Cavalieri 7, 56127 Pisa, Italy;

and INAF - Oss. Astron. Padova, vicolo dell'Osservatorio, 5, 35122 Padova, Italy

D. Arnett,

Steward Observatory, University of Arizona, 933 North Cherry Avenue, Tucson, AZ 85721, USA

D. Kagan,

Department of Astronomy, University of Texas at Austin, Austin, TX 78712, USA

A. V. Filippenko,

Department of Astronomy, University of California, Berkeley, CA 94720-3411, USA

W. Li,

Department of Astronomy, University of California, Berkeley, CA 94720-3411, USA

I. Arcavi,

Department of Particle Physics and Astrophysics, Faculty of Physics, The Weizmann Institute of Science, Rehovot 76100, Israel

S. B. Cenko,

Department of Astronomy, University of California, Berkeley, CA 94720-3411, USA

D. B. Fox,

Dept. of Astronomy and Astrophysics, Pennsylvania State University, University Park, PA 16802, USA

D. C. Leonard,

Department of Astronomy, San Diego State University, San Diego,California 92182, USA

D.-S. Moon,

Department of Astronomy and Astrophysics, University of Toronto, 50 St. George Street, Toronto, ON M5S 3H4, Canada D. J. Sand,

Harvard-Smithsonian Center for Astrophysics, 60 Garden Street, Cambridge, MA 02138, USA

and Las Cumbres Observatory Global Telescope Network, 6740 Cortona Dr., Suite 102, Goleta, CA 93117, USA

A. M. Soderberg,

Harvard-Smithsonian Center for Astrophysics, 60 Garden Street, Cambridge, MA 02138, USA

J. P. Anderson,

Departamento de Astronoma, Universidad de Chile, Camino El Observatorio 1515, Las Condes, Santiago, Casilla 36-D, 


\section{Chile;}

and Astrophysics Research Institute, Liverpool John Moores University, Twelve Quays House, Birkenhead CH41 1LD, UK

P. A. James,

Astrophysics Research Institute, Liverpool John Moores University, Twelve Quays House, Birkenhead CH41 1LD, UK

R. J. Foley,

Clay Fellow, Harvard-Smithsonian Center for Astrophysics, 60 Garden Street, Cambridge, MA 02138, USA

M. Ganeshalingam,

Department of Astronomy, University of California, Berkeley, CA 94720-3411, USA

E. O. Ofek,

Department of Astronomy, 105-24, California Institute of Technology, Pasadena, CA 91125, USA

L. Bildsten,

Kavli Institute for Theoretical Physics, Kohn Hall, University of California, Santa Barbara, CA 93106, USA and Department of Physics, University of California, Santa Barbara, CA 93106, USA

G. Nelemans,

Department of Astrophysics, Radboud University Nijmegen, P.O. Box 9010, NL-6500 GL, The Netherlands K. J. Shen,

Kavli Institute for Theoretical Physics, Kohn Hall, University of California, Santa Barbara, CA 93106, USA

N. N. Weinberg,

Department of Astronomy, University of California, Berkeley, CA 94720-3411, USA

B. D. Metzger,

Department of Astronomy, University of California, Berkeley, CA 94720-3411, USA

A. L. Piro,

Department of Astronomy, University of California, Berkeley, CA 94720-3411, USA

E. Quataert,

Department of Astronomy, University of California, Berkeley, CA 94720-3411, USA

M. Kiewe,

Department of Particle Physics and Astrophysics, Faculty of Physics, The Weizmann Institute of Science, Rehovot 76100 , Israel

and D. Poznanski,

Department of Astronomy, University of California, Berkeley, CA 94720-3411, USA

and Lawrence Berkeley National Laboratory, 1 Cyclotron Road, Berkeley, CA 94720, USA 
Supernovae (SNe) are thought to arise from two different physical processes. The cores of massive, short-lived stars undergo gravitational core collapse and typically eject a few solar masses during their explosion. These are thought to appear as as type Ib/c and II SNe, and are associated with young stellar populations. A type Ia SN is thought to arise from the thermonuclear detonation of a white dwarf star composed mainly of carbon and oxygen, whose mass approaches the Chandrasekhar limit[1, 2]. Such SNe are observed in both young and old stellar environments. Here we report our discovery of the faint type Ib SN 2005E in the halo of the nearby isolated galaxy, NGC 1032. The lack of any trace of recent star formation near the SN location (Fig. 1), and the very low derived ejected mass $\left(\sim 0.3 \mathbf{M}_{\odot}\right)$, argue strongly against a core-collapse origin for this event. Spectroscopic observations and the derived nucleosynthetic output show that the SN ejecta have high velocities and are dominated by helium-burning products, indicating that SN 2005E was neither a subluminous [3, 4] nor a regular[1] SN Ia (Fig. 2). We have therefore found a new type of stellar explosion, arising from a low-mass, old stellar system, likely involving a binary with a primary white dwarf and a helium-rich secondary. The SN ejecta contain more calcium than observed in any known type of SN and likely additional large amounts of radioactive ${ }^{44} \mathrm{Ti}$. Such SNe may thus help resolve fundamental physical puzzles, extending from the composition of the primitive solar system and that of the oldest stars, to the Galactic production of positrons. 
We discovered a supernova (SN) explosion (SN 2005E; Fig. 1) on Jan. 13, 2005 (UT dates are used throughout this paper) shortly after it occurred (it was not detected on Dec. 24, 2004). Follow-up spectroscopy (Fig. 2) revealed strong lines of helium and calcium, indicating that it belongs to the previously identified group of calcium-rich type $\mathrm{Ib} \mathrm{SNe}[5]$. The SN position is $\sim 22.9 \mathrm{kpc}$ (projected) from the centre and $\sim 11.3 \mathrm{kpc}$ above the disk of its edge-on host galaxy, NGC 1032 (Fig. 1), which is itself at a distance of $34 \mathrm{Mpc}$. NGC 1032 is an isolated galaxy [6] showing no signs of interaction, with the closest small satellite galaxy found at a distance $>120 \mathrm{kpc}$ in projection. Deep follow-up observations of the explosion site, sensitive to both ultraviolet light from hot young stars and emission lines from ionized hydrogen gas, put strict limits on any local star-formation activity at or near the SN location (Fig. 1). In addition, a radio signature, expected from some core-collapse SNe, has not been observed (see Supplementary Information; SI, Section 2).

Our analysis of the spectra of SN 2005E indicates that it is similar to SNe Ib (Fig. 2 and SI, Section 3), showing lines of He but lacking either hydrogen or the hallmark Si and $\mathrm{S}$ lines of SNe Ia in its photospheric spectra. The nebular spectrum of this event shows no emission from iron-group elements, which also characterize type Ia SNe (SI, Sections 3 and 4). Analysis of this spectrum indicates a total ejected mass of $\mathrm{M}_{\mathrm{ej}} \approx 0.275$ $\mathrm{M}_{\odot}$, with a small fraction in radioactive nickel, consistent with the low luminosity of this event. Such low ejecta mass for a SN of any type has never before been firmly established using nebular spectral analysis (SI, Fig. S3 and Section 5). We also used the narrow, fast, and faint light curve (SI, Fig. S4 in Section 6) together with the measured ejecta velocity $\left(\sim 11,000 \mathrm{~km} \mathrm{~s}^{-1}\right)$ to infer the ejected mass (SI, Section 6$)$. We use these data to find consistent results of $M_{\mathrm{ej}} \approx 0.3 \pm 0.1 \mathrm{M}_{\odot}$, assuming that some of the mass is not accounted for by the nebular spectrum analysis (e.g., high-velocity He layers and some slowly moving, denser ejecta that are still hidden below the photosphere at that time). Finally, SN 2005E exhibits a remarkable amount of calcium in its ejecta, $0.135 \mathrm{M}_{\odot}(\sim 0.49$ of the total ejecta mass), 5-10 times more than typical SNe of any variety, with a relative calcium fraction 25-350 times higher than any reported values for other SNe (see Table 1 and SI, Section 7), while not showing evidence for sulfur (SI, Section 4).

The remote position of SN 2005E in the outskirts (halo) of the galaxy, together with the isolation of NGC 1032 and its classification as an S0/a galaxy (in which the starformation rate is very low[7]), in addition to our limits on local star formation, point to a SN progenitor from an old stellar population (see also SI, Section 2). In addition, the low ejected mass and nucleosynthetic output of SN 2005E are in stark contrast to those expected from collapsing massive stars, whether formed locally or ejected from a distant location (SI, Sections 8-9).

The low ejected mass is also inconsistent with those determined for SNe Ia, restricted to a tight mass range of $\sim 1-1.3 \mathrm{M}_{\odot}$, regardless of their intrinsic luminosity (even the prototype faint SN 1991bg is found in this range) 2]. Furthermore, the light curve of SN 2005E (see SI, Section 6) shows a different behavior than that of SNe Ia, declining 
much faster than even the most subluminous (SN 1991bg-like) events observed[8]. These properties, together with the observed He-rich spectra and inferred composition, rule out SN 2005E as being either a regular or peculiar SN Ia (see also discussion in the SI, Section 10, regarding the very subluminous SN 2008ha and other related peculiar SNe 4, 9, 10]). Therefore, we conclude that SN 2005E is the first clearly identified example of a new, different type of SN explosion, arising from a He-rich, low-mass progenitor.

The spectroscopic signatures of SN 2005E are quite unusual, and allow one to identify additional similar events [5]. Arising from lower-mass progenitors, these events are likely to be found among both old and young stellar populations - that is, we expect to find such peculiar SNe Ib in both early- and late-type galaxies. Indeed, while the unusual location of SN 2005E triggered the current study, several other calcium-rich subluminous SNe Ib/c similar to SN 2005E have been observed (SI, Section 11). Of the group of eight subluminous calcium-rich $\mathrm{SNe} \mathrm{Ib} / \mathrm{c}$ identified (seven identified by us and an additional one described in Ref. [11]), four are observed in old-population environments: SN 2005 E presented here, as well as SNe 2000ds, 2005cz, and SN 2007ke, residing in elliptical galaxies. SN 2000ds has pre- and post-explosion Hubble Space Telescope images showing no evidence for either star-forming regions or massive stars[12] near its location. The host-galaxy distribution of the SNe in our sample (Fig. 3) is inconsistent with that of any core-collapse SN. No radio signatures have been observed either (see SI, Section 11). Thus, all evidence suggests that a well-defined subset of SNe Ib, all having Ca-rich spectra and faint peak magnitudes, comprise a distinct physical class of explosions coming from low-mass, old progenitors. This class includes all known type Ib/c events in confirmed elliptical galaxies [13, 14] (SI, Section 11). A different interpretation[11, invoking the core collapse of a massive progenitor, was suggested for one of these events (SN 2005cz). It is difficult to reconcile our observations and analysis of SN 2005E with such an interpretation, which is also inconsistent with the host-galaxy distribution of all of the other Ca-rich $\mathrm{SNe} \mathrm{Ib}$ in our sample.

Calcium-rich SNe were theoretically predicted to arise from burning helium-rich material on a WD (e.g., a helium WD or a helium star accreting onto a CO WD), leading to the full disruption of a sub-Chandrasekhar-mass WD[15, 16]. However, such models predicted the production of SNe far more luminous (and ${ }^{56} \mathrm{Fe}$ rich) than SN 2005E. Several theoretical models were suggested in the literature to possibly produce subluminous SNe, with low-mass and high-velocity ejecta in an old stellar population. These include the accretion-induced collapse (AIC) of a WD (e.g., Refs. [17] and [18]), and the detonation of an accreted helium shell on a WD in a binary system (the "Ia" model[19]). These studies did not explore the burning of large helium masses $\left(>0.1 \mathrm{M}_{\odot}\right)$, nor the production of calcium-rich ejecta. Multi-dimensional simulations of a detonation in accreted He layers [20] showed (for low-mass white dwarfs; $M=0.7 \mathrm{M}_{\odot}$ ) a trend toward large Ca abundances and high $\mathrm{Ca} / \mathrm{S}$ abundance ratio (a high ratio is inferred for SN 2005E; see SI, Section 4) and a light curve that was faster and dimmer than those of typical SNe Ia (but still much more luminous than SN 2005E), as well as a high production of ${ }^{44} \mathrm{Ti}$. It 
is possible that similar models, with less burning of $\mathrm{C}$ and $\mathrm{O}$ to make $\mathrm{S}$ and $\mathrm{Ni}$, may resemble SN 2005E. This gains additional support from our nucleosynthetic analysis (see SI, Section 12), showing that the unique composition of SN 2005E could be produced, in principle, as the product of He ignition. Further studies in these directions are in progress.

We conclude that SN 2005E appears to be the first observed manifestation of the helium detonation process. This event most likely occurred in an interacting double WD system with a helium WD mass donor. Additional characteristics of these explosions, including their old population origin, He-rich spectra, subluminosity, and low ejected mass, are broadly consistent with the predictions of some theoretical models (.Ia[19]; AIC[18]; helium detonation[21]), variants of which may produce the appropriate conditions for such helium detonations. Alternatively, these explosions may require a totally new mechanism.

Our discovery has numerous astrophysical implications. It seems highly likely that we identified explosions arising from very close WD-WD systems, the rates of which (SI, Section 11) might be useful for predicting the rates of WD-WD inspirals observable as gravitational wave sources. The unique nucleosynthetic production of large masses of calcium and radioactive ${ }^{44} \mathrm{Ti}$ per explosion could solve puzzles related to the source of calcium (especially ${ }^{44} \mathrm{Ca}$ ) in the primitive solar system[22, 23] and in old, metal-poor halo stars 24], and the enrichment patterns of the interstellar and intracluster medium 25]. Production of most of the Galactic ${ }^{44} \mathrm{Ti}$ and its progeny, ${ }^{44} \mathrm{Ca}$, in a few rare, prolific explosions, can also explain the origins of Galactic ${ }^{44} \mathrm{Ca}$ given the null detection of ${ }^{44} \mathrm{Ti}$ traces in most nearby SN remnants[23, 26].

Finally, inverse $\beta$ decay of ${ }^{44} \mathrm{Ti}$ may significantly contribute to the Galactic production of positrons 27]. Assuming our estimated rates ( $\sim 10 \%$ of the SN Ia rate; SI, Section 11 ) and our ${ }^{44} \mathrm{Ti}$ yield $\left(0.014-0.14 \mathrm{M}_{\odot}\right.$; SI, Section 12), Galactic SNe of the type we describe here will provide a significant contribution to the Galactic bulge component of the positron annihilation line, at least comparable to that of SNe Ia. In fact, within the current uncertainties on the ${ }^{44} \mathrm{Ti}$ yield and $\mathrm{SN}$ rates, these events may come within a factor of a few of producing all of the observed positrons 28 . 
We would like to thank P. Podsiadlowski, E. Nakar, D. Maoz, and the referees for helpful comments. We acknowledge observations with the Liverpool Telescope, and various telescopes at the Lick, Palomar, and Keck Observatories. We are grateful to the staffs of these observatories, as well as to the institutions, agencies, and companies funding these facilities. This research has also made use of the NASA/IPAC Extragalactic Database (NED). H.B.P. acknowledges the ISF/FIRST and Ilan Ramon-Fulbright Fellowships. The collaborative work of A.G. and P.M. is supported by a Weizmann-Minerva grant. A.G. acknowledges further support by the Israeli Science Foundation, an EU Seventh Framework Programme Marie Curie IRG Fellowship, the Benoziyo Center for Astrophysics, and the Peter and Patricia Gruber Awards. A.V.F. is grateful for the support of the US National Science Foundation, the US Department of Energy, Gary and Cynthia Bengier, the Richard and Rhoda Goldman Fund, the Sylvia \& Jim Katzman Foundation, and the TABASGO Foundation.

H.B.P. led the project, performed the calculations related to hyper-velocity stars, examined other putative SN 2005E-like events, collected and analyzed archival data concerning SN properties and their hosts, and wrote the manuscript. A.G.-Y. is the PI of the CCCP program and initiated the project, collected and analyzed photometric and spectroscopic data, coordinated further observational and theoretical work, and managed the project. P.A.M. conducted the nebular spectral analysis and its interpretation, and determined the elemental abundances in the ejecta. D.A. determined that the measured composition requires He burning and performed nucleosynthesis calculations to confirm this. D.K. investigated local star-formation tracers at the location of SN 2005E. A.V.F. and W.L. contributed spectroscopic and photometric observations and reductions of SN 2005E and of similar Ca-rich objects, a class they originally identified, and provided most of the data on SN host galaxies. A.V.F. also carefully edited the paper. I.A. analyzed the CCCP photometry of SN 2005E and cross-calibrated it with other data. S.B.C., D.B.F., D.C.L., D.-S.M., D.J.S., and A.M.S. are members of the CCCP and contributed to initial observations of SN 2005E. J.P.A. and P.A.J. obtained and analyzed narrow-band images of NGC 1032 and the location of SN 2005E. R.J.F. and M.G. contributed to spectroscopic observations and reductions. E.O.O. obtained deep photometric observations of the location of SN 2005E. L.B., G.N. K.J.S., and N.N.W. investigated the relation of SN 2005E to .Ia models and contributed to the text. B.D.M., A.L.P., and E.Q. investigated the relation of SN 2005E to AIC models and contributed to the text. M.K. performed custom reductions of CCCP spectra. D.P. carried out synthetic photometry analysis.

The authors declare no competing financial interests.

Correspondence should be addressed to A. Gal-Yam (avishay.gal-yam@weizmann.ac.il) and H. B. Perets (hperets@cfa.harvard.edu). 
Supplementary Information accompanies the paper on www.nature.com/nature

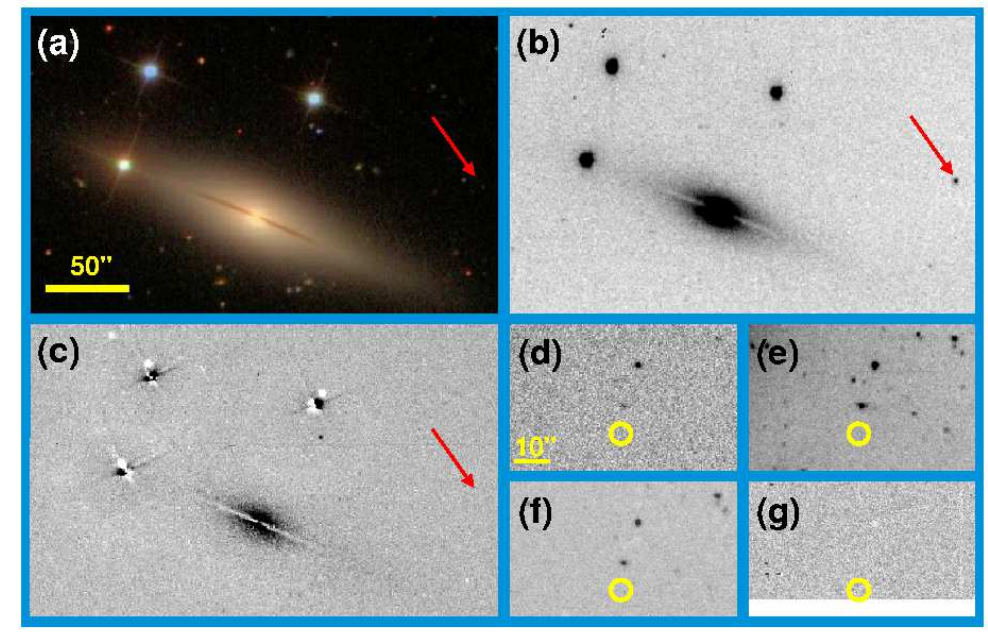

Figure 1: The environment of SN 2005E (technical details about the observations can be found in SI, Section 1). (a) NGC 1032, the host galaxy of SN 2005E, as observed by the Sloan Digital Sky Survey (SDSS), prior to the SN explosion. The galaxy is an isolated, edge-on, early-type spiral galaxy, showing no signs of star-formation activity, warping, or interaction. Its luminosity is dominated by the cumulative contribution of a multitude of low-mass old stars (yellow light in this image). Panels (a)-(c) are $275^{\prime \prime} \times 175^{\prime \prime}$; a scale bar is provided, north is up, and east is to the left. (b) The LOSS[29] discovery of SN 2005E on Jan. 13, 2005 (shown in negative). Note the remote location of the SN (marked with a red arrow) with respect to its host, $22.9 \mathrm{kpc}$ (projected) from the galaxy nucleus and $11.3 \mathrm{kpc}$ above the disk, whose edge-on orientation is well determined (panel (a)). (c) An image of NGC 1032 in the light of the $\mathrm{H} \alpha$ emission line, emitted by interstellar gas ionized by ultraviolet (UV) radiation, and a good tracer of recent star formation. There are no traces of recent star-formation activity (usually appearing as irregular, compact emission sources) near the SN location or anywhere else in the host. Panels (d)-(g) are $64^{\prime \prime} \times 36^{\prime \prime}$; a scale bar is provided. (d) Zoom-in on the location of SN $2005 \mathrm{E}$ in pre-explosion SDSS $r$-band images. No source is detected near the SN location, marked with a yellow circle (radius $3^{\prime \prime}$; the astrometric uncertainty in the SN location is $<0.5^{\prime \prime}$ ). The SDSS catalog does not list any objects near that position (e.g., putative faint dwarf satellites of NGC 1032), down to a typical limit of $r=22.5$ mag. (e-f) Deeper photometry of the SN location. A red image is shown in panel (e), while a UV (u-band) 
image is shown in panel (f). At the distance of NGC 1032, the point-source upper limits we find, $M_{r}<-7.5(-6.9)$ and $M_{u^{\prime}}<-8.1(-7.1) \mathrm{mag}$ at $3(2) \sigma$, respectively, indicate that we would have detected faint star-forming galaxies or star-forming regions at the SN location, or indeed even individual massive red supergiant or luminous blue supergiant stars. (g) Zoom-in on the location of SN 2005E in $\mathrm{H} \alpha$ light (see panel (c) for details). No trace of star-formation activity is seen near the SN location. 

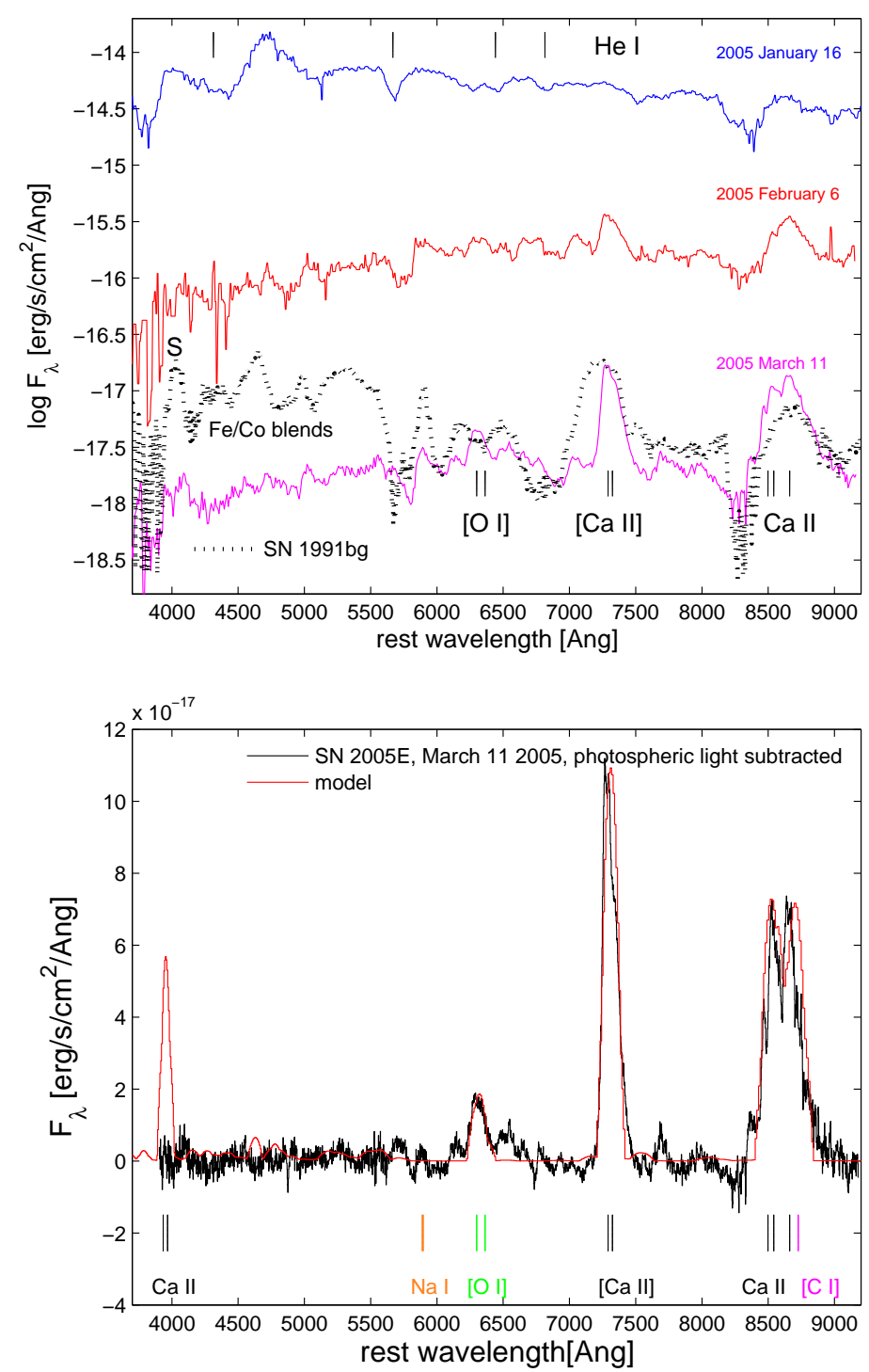

Figure 2: The mass and composition of the SN 2005E ejecta (technical details of observations and additional references can be found in SI, Section 1). Upper panel: Photospheric spectra of SN 2005E. The top spectrum is obviously photospheric and shows absorption lines of the He I series (marked with black ticks after application of an 11,000 $\mathrm{km} \mathrm{s}^{-1}$ blueshift, at the top). Nebular lines of intermediate-mass elements, most notably calcium, begin to emerge in the middle spectrum. Calcium dominates the latest nebular spectrum at the bottom, and nebular oxygen is visible as well. Also note that the typical Si lines of SNe Ia are absent in all spectra, while the nebular spectrum of SN 2005E clearly rules out a type Ia identification (comparison with the underluminous SN 1991bg is shown; note the lack of the typical iron-group line blends in the blue side). The derived line velocities are consistent with SN 2005E exploding within its putative host galaxy, 
NGC 1032. Bottom: The nebular spectrum of SN 2005E compared with a model fit. From the fit we can derive elemental abundances and masses in the ejecta of SN 2005E. We find masses of $0.1,0.037,0.135$, and $0.003 \mathrm{M}_{\odot}$ for carbon, oxygen, calcium, and radioactive nickel, respectively. Both the low total ejected mass of $\sim 0.275 \mathrm{M}_{\odot}$ and the relative abundances are unique among previously studied events. The lack of prominent C/O-burning products such as S and Fe (typically seen in SNe Ia; SI, Section 4) argues against a $\mathrm{C} / \mathrm{O} \mathrm{WD}$ origin. 


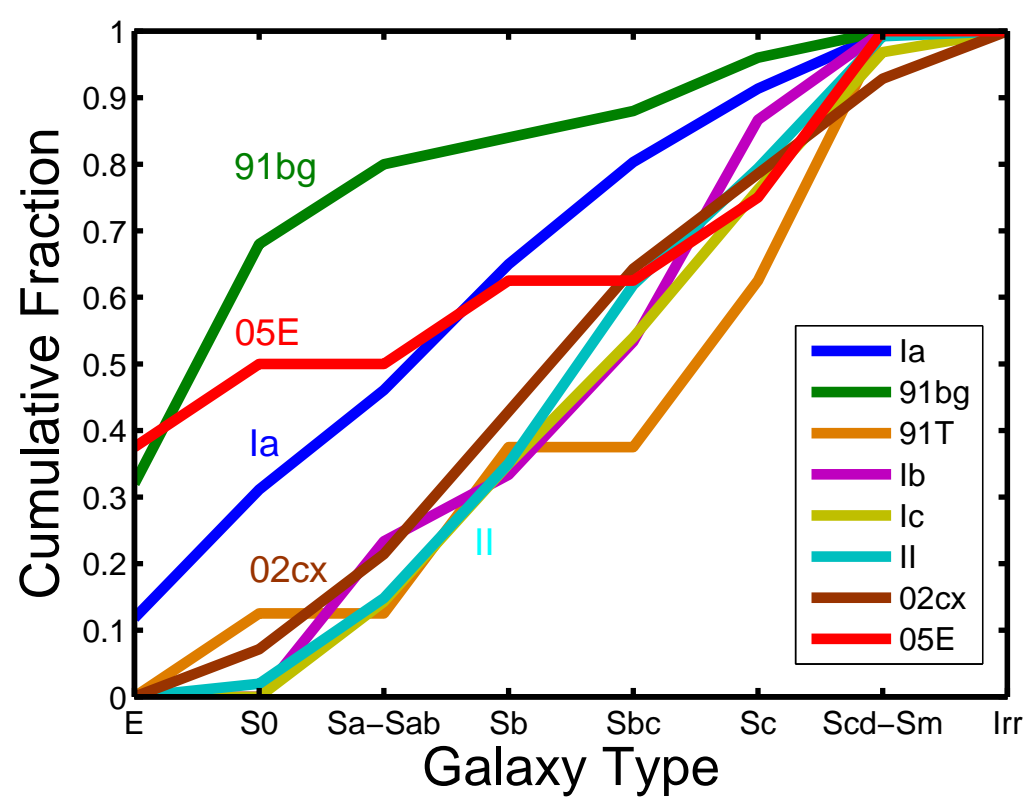

Figure 3: The cumulative distribution of host galaxies of SNe from the KAIT SN survey. We corrected the classification of a few SN Ib/c hosts using higher-quality observations from the Palomar 60-inch telescope (SN 2005ar, 2006ab, and 2006lc were found to be hosted by spiral galaxies rather than elliptical galaxies). After correcting the classification we find that all $\mathrm{SNe} \mathrm{Ib} / \mathrm{c}$ found in early-type galaxies are faint Ca-rich SNe similar to SN 2005E. Note that the SN 2005E-like SN host distribution is very different from that of other $\mathrm{SNe} \mathrm{Ib} / \mathrm{c}$, as well as that of SNe II (known to have young massive progenitors) and that of SN 2002cx-like SNe Ia, with half of the SN 2005E-like group (four out of eight) observed in early-type (elliptical or S0) galaxies. The progenitors of SN 2005E and the other members of its group are therefore likely to belong to an old, low-mass stellar population. The total numbers of host galaxies included in this figure are 244, 25, 8, 257, 30, 63, 14, and 8 for SNe of types Ia, 91bg, 91T, II, Ib, Ic, 02cx, and 05E, respectively. 


\section{$\underline{\text { Supplementary Information }}$}

\section{(1) Technical observational details for figures 1 and 2}

Figure 1 Panel (a) shows the image of NGC 1032 prior to explosion of SN 2005E, obtained from the Sloan Digital Sky Survey (SDSS) archive. Panel (b) shows the LOSS 29] discovery of SN 2005E on Jan. 13, 2005. LOSS imaging of SN 2005E was obtained using the robotic 76-cm Katzman Automatic Imaging Telescope (KAIT) at Lick Observatory. Panel (c) shows an image of NGC 1032 in the light of the $\mathrm{H} \alpha$ emission line; the panel shows the difference between images obtained using a narrow filter $(6567 \AA$; full width at half maximum $\sim 100 \AA$ ) with a measured transmission of $\sim 40 \%$ for $\mathrm{H} \alpha$ at the redshift of NGC 1032, and broad $R$-band observations used for continuum subtraction. The images, with exposure times of $1800 \mathrm{~s}$, were obtained on Oct. 5, 2008 using the RATCam camera mounted on the 2-m Liverpool Telescope at Observatorio del Roque de Los Muchachos (La Palma, Spain). The smooth negative residual ( $\sim 7 \%$ of the original flux) near the galaxy core probably arises from a combination of slight color gradients of the smooth galactic old population, and $\mathrm{H} \alpha$ absorption in the spectra of old stars, and does not indicate real line emission. Panel (d) shows a zoom-in on the location of SN 2005E in pre-explosion SDSS $r$-band images. Panels (e)-(f) show deep photometry of the SN location obtained using the Low-Resolution Imaging Spectrometer (LRIS) [30] mounted on the Keck-I 10-m telescope on Feb. 17, 2009 under very good conditions (seeing $\sim 0.7^{\prime \prime}$ ). Panel (e) shows a red image with a total exposure time of $840 \mathrm{~s}$, reaching a point-source detection limit of $r<25.3(25.9) \mathrm{mag}$ at 3(2) $\sigma$. Panel (f) shows a UV ( $u$-band) image with a total exposure time of $780 \mathrm{~s}$, reaching a point-source detection limit of $u<24.7(25.7)$ mag at $3(2) \sigma$. Panel (g) shows a zoom-in on the location of SN 2005E in $\mathrm{H} \alpha$ light from the same observations used to produce panel (c).

Figure 2 Upper panel: Photospheric spectra of SN 2005E. The top two spectra were obtained as part of the Caltech Core-Collapse Project (CCCP) 31] using the double-beam spectrograph [32] mounted on the 5-m Hale telescope at Palomar Observatory. Exposure times were $600 \mathrm{~s}$ and $900 \mathrm{~s}$ on 2006 January 16 and February 6, respectively, with the 158 lines $\mathrm{mm}^{-1}$ and 1,200 lines $\mathrm{mm}^{-1}$ gratings, yielding an instrumental resolution of $\sim 5 \AA$ and $\sim 0.5 \AA$ on the red and blue sides, respectively. The CCCP spectra were further rebinned to $\sim 5 \AA$ resolution bins to increase the signal-to-noise ratio. The bottom spectrum was obtained using LRIS[30] mounted on the Keck I 10-m telescope on 2005 March 11. We took an exposure of $600 \mathrm{~s}$ using the 560 dichroic and the 400/8500 grating and 600/4000 grism, giving resolutions of $5.6 \AA$ and $2.4 \AA$ in the red and blue sides, respectively. For comparison, we plot a nebular spectrum of SN 1991bg; see Ref. [33] for a detailed discussion.

Note that the nebular spectrum contains residual photospheric light. We have addressed the issue of disentangling the photospheric and nebular components of late-time spectra (in order to use the nebular part for abundances analysis; Figs. S1, S2) following 
the approach used in the similar case of SNe 1997ef and 1997dq[34].

Our method of choice was to use the spectral-fitting code Superfit 35] to find the best match to the late-time spectrum of SN $2005 \mathrm{E}$ with the fit limited to a spectral range $(\lambda<6200 \AA)$ which is free from strong nebular emission lines. The best-fit spectrum was that of the type Ic SN 1990U, 41 days past discovery, which provides an excellent fit to the photospheric component (Fig. S1) across the observed spectrum including the wavelength ranges not included in the fit. We then replaced areas in the best-fit SN 1990U template spectrum affected by residual host-galaxy narrow $\mathrm{H} \alpha$ line contamination by a linear interpolation, and subtracted the resulting photospheric "best-fit spectrum" from the SN 2005E data to get a clean nebular-only spectrum, which was then used in the nebular-modeling analysis

We note that alternative photospheric subtraction methods (e.g., wavelength-unconstrained Superfit modeling or simple low-order polynomial continuum fits) give very similar results. 


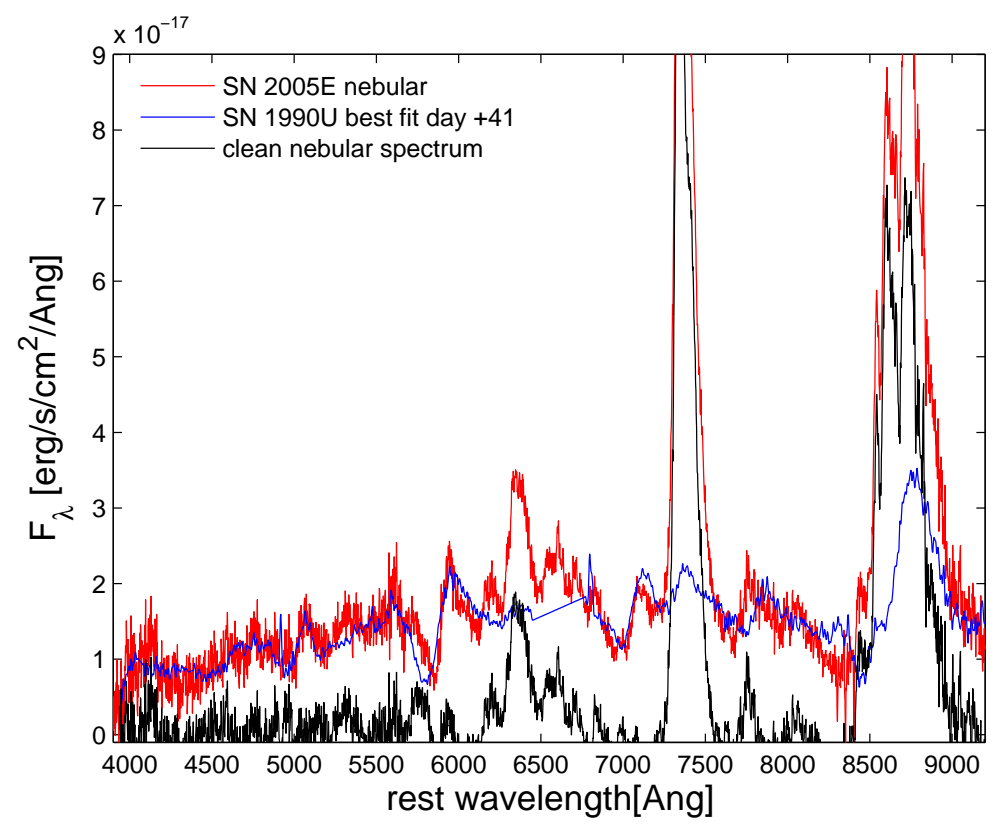

Figure S1:

Fitting the photospheric component of the late-time spectrum of SN 2005E. We show the best-fit photospheric template (SN 1990U 41 days past discovery, blue) superposed on the original SN 2005E spectrum (red); note the excellent agreement outside the range of the strong nebular $\mathrm{Ca}$ and $\mathrm{O}$ lines. The final clean nebular spectrum (black) was obtained by subtracting the best-fit template (blue) from the data (red) after interpolating over the $\mathrm{H} \alpha$ region in the template, contaminated by narrow host-galaxy lines. 


\section{(2) Can the progenitor of SN 2005E be a massive star formed in a star-forming region in the halo?}

Massive stars are typically formed and observed in giant molecular clouds and young stellar clusters or associations [36, 37]. Core-collapse SNe from massive stars are therefore expected to be found close to star-forming regions. Observations of such SNe are usually consistent with this picture 38. In principle, the discovery of SN 2005E in the halo of NGC 1032 could be attributed to in-situ star formation of a massive star rather than a low-mass older progenitor. However, star formation in the halo environment of a $\mathrm{S} 0 /$ a galaxy would be difficult to understand according to current star-formation theories. For example, star formation during collisions between cloudlets within high-velocity clouds at high galactic latitudes [39] has been shown to be much too rare[40]. Spiral density waves in the disk may trigger star formation up to a kpc above the Galactic plane[41], but this seems unlikely for the larger height of SN 2005E (which also appears to be positioned beyond the edge of the optical disk). In addition, we note that NGC 1032 shows no evidence for warping or other structures extending beyond the region of the galactic disk to which SN 2005E could be related. We conclude that given the remote location of the supernova in the galactic halo, and the nondetection of any star-formation activity anywhere in the halo or the disk of NGC 1032, it is unlikely that an in-situ formation scenario could explain SN 2005E, unless a yet unknown and unique star-formation mechanism was at work in this case. In contrast, the evidence for a low-mass progenitor of SN 2005E is naturally consistent with the low-mass old stellar population environment in which it was found. Such evidence is further supported by the host-galaxy distribution of the larger sample of Ca-rich SNe we report here, which is dominated by early-type galaxies (see Fig. 3). A search for nearby star-forming regions:

We have looked for star-formation tracers both in the halo and the disk of NGC 1032. Star-forming regions produce two classes of emission: continuum emission from young stars and emission lines (dominated by $\mathrm{H} \alpha$ ) produced by ionized gas. We have searched for both classes of emission, and obtained upper limits on the star-formation rates. $\underline{\mathrm{H} \alpha}$ observations:

$\mathrm{H} \alpha$ imaging was obtained with the Liverpool Telescope, and then analyzed using similar methods to those described in detail elsewhere [38]. We have determined an upper limit of $2.02 \times 10^{-17} \mathrm{erg} \mathrm{cm} \mathrm{cm}^{-2}$ on the $\mathrm{H} \alpha$ flux from the region of SN 2005E. This is a $3 \sigma$ upper limit obtained from the variation in the sky background, for a $2^{\prime \prime}$ aperture centred on the SN position and calibrated using an $R$-band galaxy magnitude taken from the literature 42]. For the distance of NGC 1032 (34 Mpc) we infer an H $\alpha$ luminosity of $2.79 \times 10^{36} \mathrm{erg} \mathrm{s}^{-1}$; correcting this for Galactic extinction (0.098 mag) and for the contribution from [N II] lines [43, we then calculate a corrected limit of $\mathrm{H} \alpha_{\text {limit }}=2.3 \times 10^{36}$ $\operatorname{erg~}^{-1}$. Using the conversion rate from Ref. [7] (Eq. 2), we determine an upper limit on the star-formation rate $(\mathrm{SFR})$ at the $\mathrm{SN}$ position of $\mathrm{SFR}_{\text {limit }}=1.8 \times 10^{-5} \mathrm{M}_{\odot} \mathrm{yr}^{-1}$ down to our detection limits.

In addition, our $\mathrm{H} \alpha$ observations of NGC 1032 show no star-forming regions closer 
than the galactic nucleus itself (see Fig. 1; likely the origin of the $\mathrm{H}$ I detected in this galaxy [44], similar to many other such galaxies in which molecular gas is typically centrally concentrated 45]), up to our detection limit.

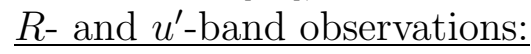

Our deep $R$ and $u^{\prime}$-band observations using Keck (calibrated onto the SDSS photometric system) rule out point sources near the location of SN 2005E down to $u^{\prime}<24.7(25.7)$ ), $r<25.3(25.9) \mathrm{mag}$ at 3(2) $\sigma$ (see Fig. 1). At the distance of NGC 1032, these limits $\left(M_{r}<-7.5(-6.9)\right.$ and $M_{u^{\prime}}<-8.1(-7.1)$ mag at $3(2) \sigma$, respectively) indicate that we would have detected faint star-forming galaxies or star-forming regions at the SN location, or indeed even individual red supergiant or luminous blue supergiant stars (although the existence of a single $<20 \mathrm{M}_{\odot}$ star cannot be ruled out). Since massive stars are usually formed and observed in young stellar clusters or associations [36, 37], the lack of nearby supergiants (either red or blue) further argues against local star-formation activity. The progenitor of SN 2005E could have been a member of an undetected globular cluster; the stellar population in such a hypothetical cluster, however, would be very old (several Gy), again indicating an old, low-mass progenitor for SN $2005 \mathrm{E}$.

Radio signature of a core-collapse $\mathrm{SN}$ :

A non-negligible fraction of core-collapse $\mathrm{SNe}$ show radio emission. We have therefore made observations at $8.46 \mathrm{GHz}$ with the VLA radio telescope on Jan. 21.10, 2005. We found a flux of $11 \pm 53 \mu \mathrm{Jy}$ at the optical position of SN 2005E. At the distance of SN 2005E, and assuming an explosion date between Dec. 24, 2004 and Jan. 14, 2005, the radio luminosity limit $(2 \sigma)$ is $1.8 \times 10^{26} \mathrm{erg} \mathrm{s}^{-1} \mathrm{~Hz}^{-1}$, which is a factor of 10 lower than a typical radio-emitting $\mathrm{SN} \mathrm{Ib} / \mathrm{c}$ on this same timescale[46].

\section{(3) Spectroscopic identification of SN 2005E as a type Ib supernova}

In Fig. 2 we show optical spectra of SN 2005E. Our first spectrum (Fig. 2 top, blue curve) is clearly photospheric, dominated by absorption lines including the He I series at 4471, 5876, 6678, and $7065 \AA$, blueshifted by $\sim 11,000 \mathrm{~km} \mathrm{~s}^{-1}$ (marked with black ticks at the top of Fig. 2), typical of a young SN Ib. Based on prediscovery nondetections, SN 2005E was 3-20 days after explosion at this time. Analysis using the Superfit spectral analysis code 35] confirms a type Ib identification, with the best-fit match being with a spectrum of the type Ib/c transition event SN 1999ex[47] 14 days after maximum light.

Our next spectrum (Fig. 2 middle, red curve) shows the beginning of the transition to the nebular phase, with emerging emission lines of calcium. The best-fit spectrum found by superfit is that of the type Ic event SN 1990U, but spectra of the type Ib SN 1999di also provide a good fit. Masking of the blueshifted He I $6678 \AA$ line by the emerging [O I] $\lambda \lambda 6300,6364$ nebular doublet may account for the similarity to SNe Ic, with intrinsically much weaker He I lines.

Strong nebular emission lines of [O I] $\lambda \lambda 6300,6364$, and especially [Ca II] $\lambda \lambda 7291,7324$ and the Ca near-infrared triplet at 8498, 8542, and $8662 \AA$ (tick marks at the bottom of Fig. 2), dominate our latest spectrum of SN 2005E (Fig. 2 bottom, magenta curve). The 
best fit found by Superfit is to the type Ib SN 1985F[48, 49] obtained 89 days after its first observation. Dominated by lines of intermediate-mass elements ( $\mathrm{O}$ and $\mathrm{Ca}$ ), the nebular spectrum of SN 2005E is similar to that of SNe Ib, though calcium is much stronger than usual for this SN type, while the lack of Fe-group emission lines in the bluer part of the spectrum rules out an identification as a SN Ia of either the normal or subluminous (SN 1991bg-like[3]) varieties.

\section{(4) The relative ejected mass of calcium and sulfur}

To constrain the relative ratio of ejected calcium to sulfur (Ca/S, used below to investigate the nucleosynthetic processes responsible for SN 2005E-like events), we ran a grid of nebular spectral models similar to those shown in Fig. 2b. However, we forced the $\mathrm{S} / \mathrm{Ca}$ fractions to be $0.16,0.37,0.55,0.74$, and 1.00 as shown in Fig. S2. As can be seen there, the data rule out ratios below $\mathrm{Ca} / \mathrm{S}=6$ (this limit is set by the uncertainty in photospheric light subtraction and blending with weak nebular $\mathrm{C}$ lines). Note that the similar excitation parameters and critical densities of [S I] $\lambda \lambda 4589,7725$ and [O I] $\lambda \lambda 6300$, 6364 (which is well detected) indicate that the lack of strong [S I] lines results from an abundance (rather than an excitation) effect. 

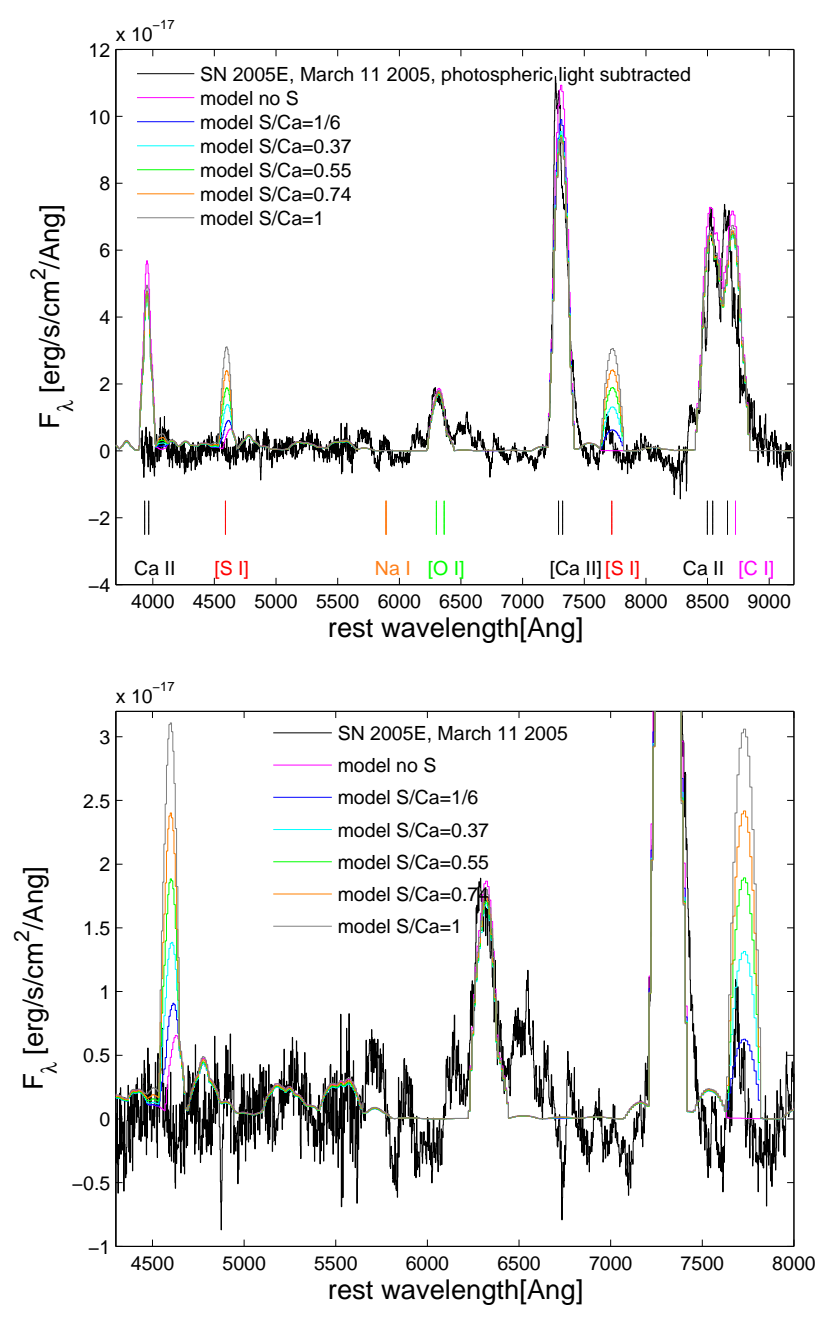

\section{Figure S2:}

An upper limit on the relative abundance of sulfur and calcium. Top: a grid of models calculated using the same input spectrum, with values of the $\mathrm{S} / \mathrm{Ca}$ ratio artificially set to $1 / 6,0.37,0.55,0.74$, and 1.00 . Note that values above $\mathrm{S} / \mathrm{Ca}=$ $1 / 6$ are clearly ruled out by the data. S lines are not detected, but lower values cannot be constrained due to uncertainty in the photospheric light subtraction and blending with nearby $\mathrm{C}$ lines. The bottom panel shows that the [O I] $\lambda \lambda 6300,6364$ line, whose excitation parameters and critical densities are similar to those of the [S I] $\lambda \lambda 4589,7725$ lines, is well detected, and thus the lack of $\mathrm{S}$ lines is a real abundance effect rather than an excitation effect. 


\section{(5) Masses and luminosities of $\mathrm{SNe}$}

The total mass, nickel mass, and luminosity of SN 2005E are far lower than those found for the majority of SNe of any type (Fig. S3). Taken together, no other SN except for SN 2008ha (see SI, Section 10 for a discussion of this SN and other related events) was both faint and has subsolar-mass ejecta. SNe Ia (excepting SN 2008ha), both regular and peculiar/subluminous, have inferred ejecta masses of $\geq \sim 1 \mathrm{M}_{\odot}[2$. SNe Ib/c and SNe II have even more massive ejecta (a few times solar) [50, 51, 52, 53, [54, 55, 56, 57]. Additional data concerning the ejected Ni mass can be found in Ref.[58]. Some SNe II-P are as faint as SN 2005E or even fainter [59], but ejected a few solar masses. 


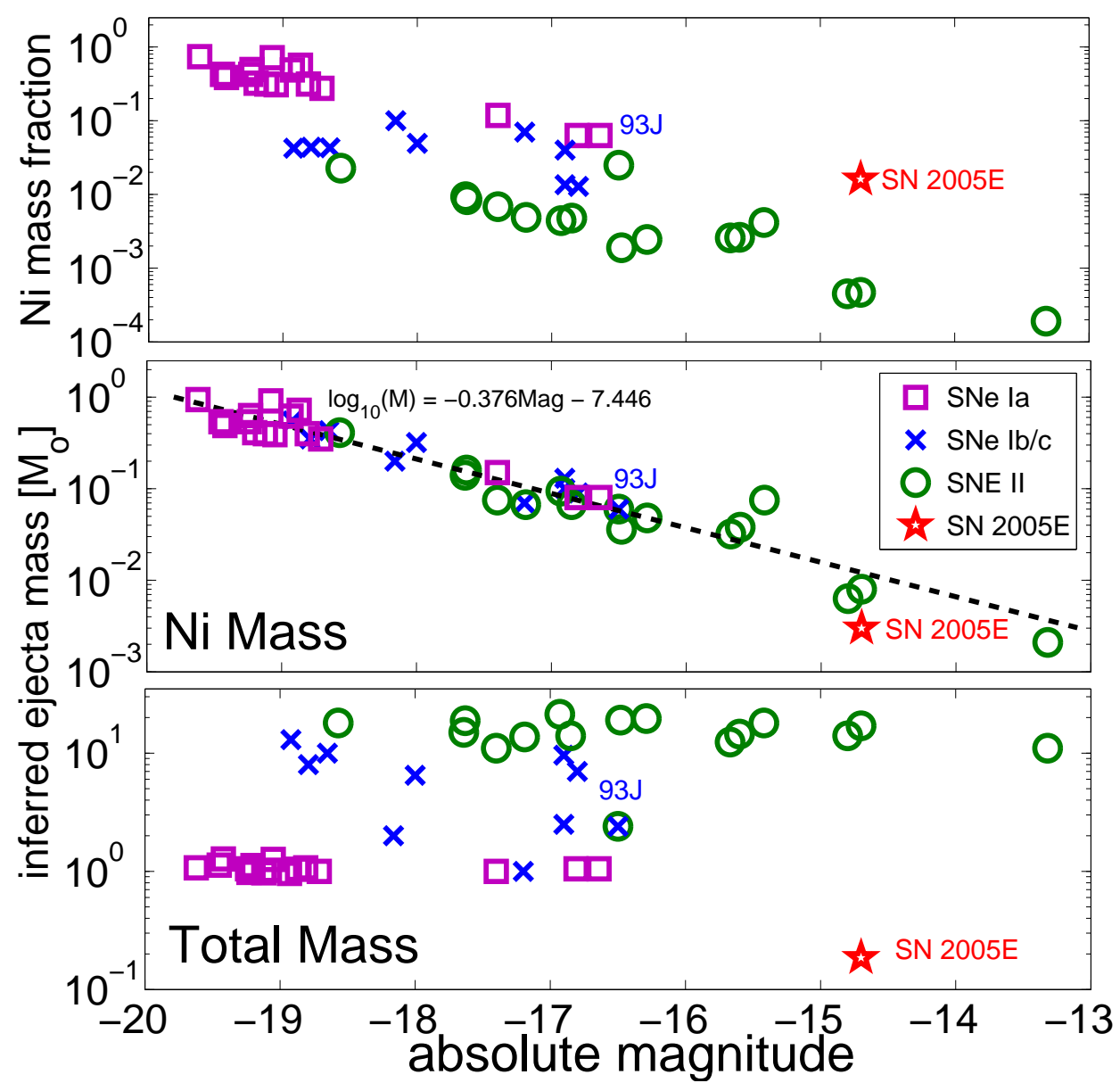

Figure S3: Comparison of the SN 2005E ejecta mass and luminosity with those of other SNe [SNe Ia, squares; $\mathrm{SNe} \mathrm{Ib} / \mathrm{c}, \times$ marks; SNe II, circles]. The lower panel shows the total ejecta mass inferred for SN 2005E, which is the lowest inferred ejecta mass found for any SN, based on nebular spectra. Its position in the luminosity vs. ejecta-mass phase space is unique, suggesting that it is not a member of the currently well-known SN families. The middle panel shows the Ni mass inferred for SN 2005E; it is small, consistent with its low luminosity, although somewhat lower than might be expected from the extension of the observed Ni mass vs. luminosity relation observed for other SNe (dashed line and formula). The upper panel shows the Ni mass fraction, $M_{\mathrm{Ni}} / M_{\text {total }}$, inferred for SN 2005E. All of the masses in this figure were inferred from detailed modeling. The error bars on the masses are $\sim 10-20 \%$; these are smaller than the symbol sizes and are not shown.

(6) Ejected mass estimates from the observed light curve and photospheric velocities

The ejecta mass of a given supernova can be estimated using its light curve and the observed ejecta velocities. The expansion velocity, $v$, of a $\mathrm{SN}$ is proportional to $\left(E_{\text {kin }} / M_{\mathrm{ej}}\right)^{1 / 2}$, where $E_{\text {kin }}$ is the kinetic energy and $M_{\mathrm{ej}}$ is the ejected mass, while the typical duration of a SN light curve is $t_{\mathrm{d}} \propto\left(M_{\mathrm{ej}}^{3} / E_{\mathrm{kin}}\right)^{1 / 4}[60$. Combining these equations 
and assuming that two objects have the same opacity, we have

$$
E_{\mathrm{kin}, 1} / E_{\mathrm{kin}, 2}=\left(\frac{v_{1}}{v_{2}}\right)^{3}\left(\frac{t_{1}}{t_{2}}\right)^{2}
$$

and

$$
M_{\mathrm{ej}, 1} / M_{\mathrm{ej}, 2}=\frac{v_{1}}{v_{2}}\left(\frac{t_{1}}{t_{2}}\right)^{2}
$$

Following Ref. [9], in which the mass of the subluminous SN 2008ha was estimated, we use a normal SN Ia as a reference with $t_{\mathrm{d}}=19.5$ days and $v=8,000 \mathrm{~km} \mathrm{~s}^{-1}[61]$. The timescales of SN 2005E are a factor of $0.45-0.55$ times those of the well-observed type Ib SN 2008D (the contracted light curve of SN 2008D is shown in Fig. S4 for comparison), which had a rise time of 18 days 62, and therefore we estimate the rise time of SN 2005E to be 79 days. The ejecta velocities we observe from the photospheric spectra are 11,000 $\mathrm{km} \mathrm{s}^{-1}$. We therefore find $E_{\text {kin,05e }} / E_{\text {kin,Ia }}=0.34-0.55$ and $M_{\text {ej,05e }} / M_{\text {ej,Ia }}=0.17-0.29$. Assuming $E_{\text {kin,Ia }}=1.3 \times 10^{51} \mathrm{ergs}$ and $M_{\mathrm{ej}, \text { Ia }}=1.4 \mathrm{M}_{\odot}$, we find $E_{\text {kin }, 05 \mathrm{e}}=(4.4-7.2) \times 10^{50} \mathrm{ergs}$ and $M_{\mathrm{ej}, 05 \mathrm{e}}=0.25-0.41 \mathrm{M}_{\odot}$. We note that this widely used method for SN ejecta-mass estimation may have two caveats in our case: helium is less opaque than other elements so we may miss some of the helium mass, and ${ }^{56} \mathrm{Ni}$ may not be the only radioactive energy source as assumed in such estimates. 

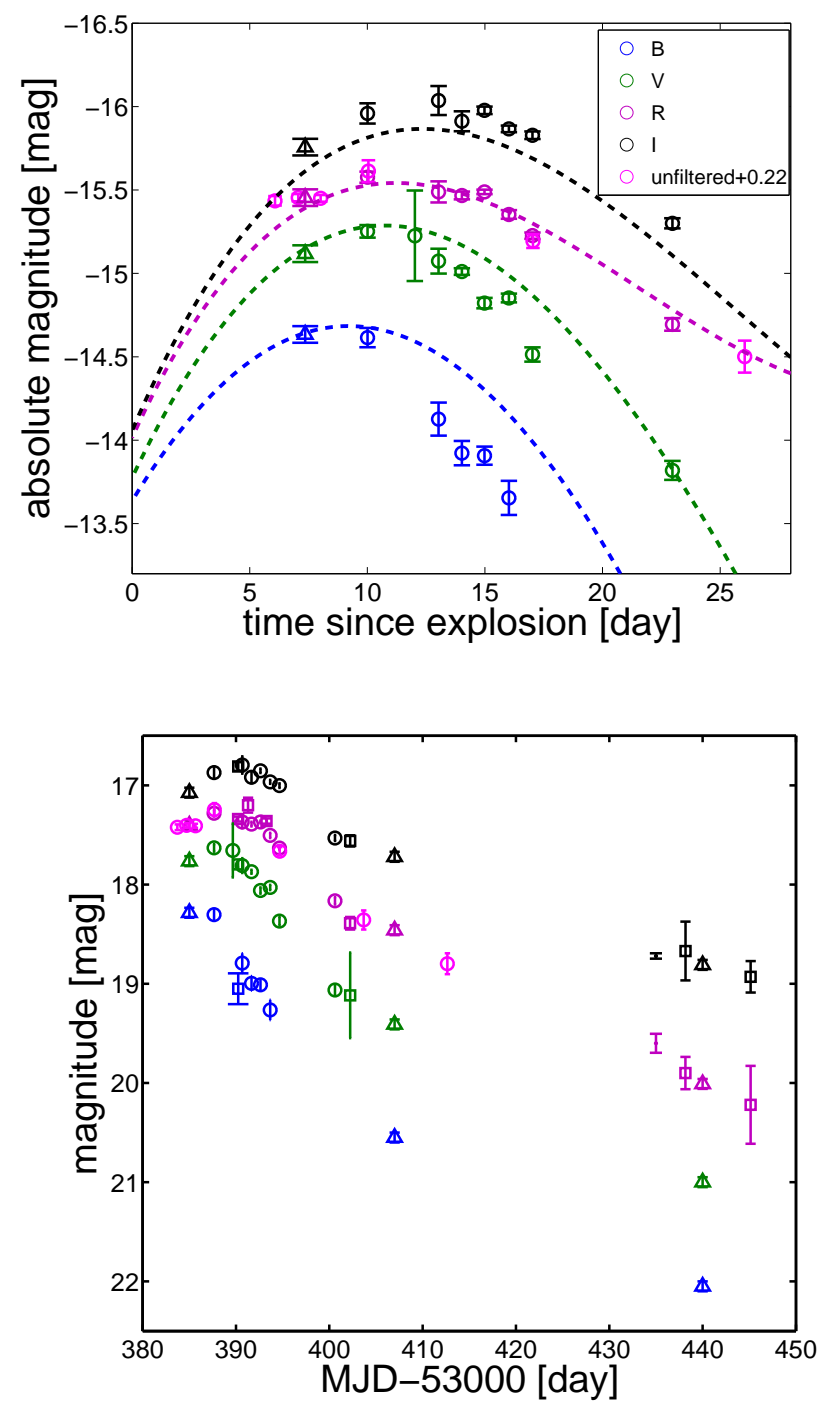

\section{Figure S4:}

Optical light curves of SN 2005E. We present our observations obtained using the 0.76-m KAIT as part of LOSS 29] in the $B V R I$ bands (blue, green, red, and black empty circles, respectively), as well as unfiltered data (magenta empty circles). Also shown are CCCP 31] BVRI observations obtained using the Palomar 60-inch telescope [P60; 63; (rectangles)] and $R I$ photometric observations with Keck (red and black points, respectively). Additional points (triangles) were obtained through synthesis of the spectra (seen in Fig. 2), scaled to fit the $R$-band photometry. KAIT unfiltered observations are most similar to $R$-band data due to the combined response of its optics and detector, so we have scaled these data to the $R$ band observations. Top: Light curve near peak. The rapid decline of this object is consistent with its relatively low absolute magnitude, $M_{R} \approx-15.5$, calculated assuming a distance of $34 \mathrm{Mpc}$ to NGC 1032 (as given in the NED database 64]) and negligible extinction. Estimating the peak date of this SN from the magenta curve (January 7, 2005), our spectra (Fig. 2) were obtained 9, 29, and 62 days after maximum light. Also shown for comparison are third-order polynomial fits to the light curves of the well-studied type Ib SN 2008D 62 time-contracted by a factor of 0.52 to fit the SN 2005E data. We find 
that SN 2005E behaves similarly to SN 2008D near peak, but with timescales multiplied by roughly 0.45-0.55. Bottom: long-term evolution of the light curve. The rapid light-curve decline is similar to that observed in subluminous SNe Ia at early times, with a slope of -0.155 mag day ${ }^{-1}$; however, it does not show a break to a different slope expected at $\sim 4.5$ days after peak (compare with the analysis of Kasliwal et al. 8]), but continues instead to decline at a nearly constant rate, even at late times. 


\begin{tabular}{|c|c|c|c|c|c|c|c|}
\hline SN (Refs.)/Element & $\mathrm{C}$ & $\mathrm{O}$ & $\mathrm{Mg}$ & $\mathrm{Si}$ & $\mathrm{S}$ & $\mathrm{Ca}$ & $\mathrm{Ni}$ \\
\hline \hline $1998 \mathrm{bw}[65]$ & $0.15(1.9)$ & $3.9(49)$ & $0.05(0.63)$ & $0.15(1.9)$ & $0.4(5)$ & $0.08(0.015)$ & $0.65(8.2)$ \\
\hline $1997 \mathrm{dq}[34]$ & $0.37(9.2)$ & $0.73(18)$ & $0.003(0.075)$ & $0.8(20)$ & $0.2(5)$ & $0.04(0.017)$ & $0.16(4)$ \\
\hline $2002 \mathrm{ap}[56]$ & $0.4(40)$ & $1.03(103)$ & $0.01(1)$ & $0.25(25)$ & $0.1(10)$ & $0.01(0.005)$ & $0.11(1.1)$ \\
\hline $2006 \mathrm{aj}[66]$ & $0.25(1250)$ & $1.42(7100)$ & $0.003(15)$ & $0.05(250)$ & $0.01(50)$ & $0.0002(0.0001)$ & $0.19(950)$ \\
\hline $1994 \mathrm{I}[55]$ & $0.11(28)$ & $0.33(83)$ & $0.003(0.75)$ & $0.1(25)$ & $0.05(12)$ & $0.004(0.006)$ & $0.08(20)$ \\
\hline $2003 \mathrm{du}[66]$ & $0(0)$ & $0.23(230)$ & $0.022(22)$ & $0.21(210)$ & $0.08(80)$ & $0.001(0.0009)$ & $0.59(590)$ \\
\hline $2004 \mathrm{eo}[67]$ & $0(0)$ & $0.43(86)$ & $0.038(7.6)$ & $0.26(52)$ & $0.04(8)$ & $0.005(0.0045)$ & $0.32(64)$ \\
\hline $1991 \mathrm{bg}[33]$ & $0(0)$ & $0.24(120)$ & $0.04(2)$ & $0.87(435)$ & $0.02(10)$ & $0.002(0.0016)$ & $0.08(40)$ \\
\hline $2005 \mathrm{hk}[68]$ & $<0.014(2.5)$ & $0.53(132)$ & - & $0.02(5)$ & $0.022(5.4)$ & $0.004(0.004)$ & $0.4(100)$ \\
\hline $2005 \mathrm{E}$ & $0.1(0.74)$ & $0.037(0.27)$ & $0(0)$ & $0(0)$ & $<0.01(0.074)$ & $0.135(0.49)$ & $0.003(0.022)$ \\
\hline
\end{tabular}

Table 1: Elemental abundances in various SNe (abundance ratios relative to Ca are shown in brackets; for Ca the ratio shown is to the total mass inferred for the SNe ejecta). This sample includes SNe of types Ib, Ic, and Ia. The SNe Ia include the prototypical subluminous SN 1991bg as well as SN 2005hk (the latter is a member of the SN 2002cx-like group). All abundances were derived using the same methods and in most cases using the same code, such that any possible systematic problems cancel out. Note that we estimate a conservative error of $<25 \%$ on the calcium abundance in SN 2005E. Specific details are given in the references for each of the $\mathrm{SNe}$.

\section{(7) Composition of SN ejecta}

Table 1 shows the elemental abundances inferred for various SNe. SN 2005E synthesized more Ca than any other known SN in the absolute sense, by typically a factor of 5-10, with the exception of massive hypernovae like SN 1998bw. Even these rare extreme events lag behind in the Ca mass fraction (a factor of 30 less than in SN 2005E) while typical SNe have Ca mass fractions of $>100$ times below that of SN 2005E, so this class indeed merits the "Ca-rich" distinction initially proposed by Ref.[5]. 


\section{(8) The possible origin of SN 2005E from a hypervelocity star and its trajec- tory}

Most of the stars in the Galaxy move at relatively low velocities (a few tens of $\mathrm{km}$ $\left.\mathrm{s}^{-1}\right)$ with respect to their Galactic environment. Some massive stars $\left(\gtrsim 8 \mathrm{M}_{\odot}\right)$ are known to have higher velocities, up to $\sim 200 \mathrm{~km} \mathrm{~s}^{-1}$. These so-called "runaway stars" are ejected from their birth place as a result of binary encounters, or if they have binary companions that explode as $\mathrm{SNe}$ 69, 70], and could therefore be found far from the starforming region where they were formed. Hypervelocity stars[71] (HVSs) move at even higher velocities $\left(\mathrm{few} \times 10^{2}-10^{3} \mathrm{~km} \mathrm{~s}^{-1}\right)$. Such stars are thought to be ejected following dynamical interaction with (or close to) massive black holes.

In 2005, the first HVS was serendipitously discovered in the Galactic halo 72, $71 \mathrm{kpc}$ from the Galactic centre (GC), with a radial velocity of $853 \pm 12 \mathrm{~km} \mathrm{~s}^{-1}$. Two additional HVSs, one of them a massive B star $\left(\sim 9 \mathrm{M}_{\odot}\right)$, were discovered shortly thereafter [73, 74]. Follow-up surveys have discovered a total of $\sim 20 \mathrm{HVSs}$ with radial velocities in the range $300-900 \mathrm{~km} \mathrm{~s}^{-1}$ at distances of $20-120 \mathrm{kpc}$ from the GC. A total population of $\sim 100$ such young B stars is inferred to exist in the Galaxy at these distances (no main-sequence O stars are observed). Given their positions and velocities, all observed HVSs must have been ejected with even higher initial velocities $\left(>850 \mathrm{~km} \mathrm{~s}^{-1}\right)$, if they originated near the massive black hole $(\mathrm{MBH})$ in the centre of our Galaxy whose mass is $\sim 3.6 \times 10^{6}$ $\mathrm{M}_{\odot}[75$, 76].

If the progenitor of SN 2005E was a massive star, it may have been formed in the centre or in the disk of NGC 1032, and was later ejected at high velocity, traveling to its observed location in the halo. In order to find the ejection velocity required for the progenitor of SN 2005E to travel from its birth place to the observed position of SN 2005E, we must trace its possible trajectory. For this purpose we need to assume some galactic potential for NGC 1032 as well as the mass for the MBH in its nucleus.

For the potential of NGC 1032, we use a two-component model suggested in Ref. [77] composed of a galactic bulge and a halo (the disk component has a relatively small effect). The bulge mass could be estimated from the velocity dispersion in the bulge[78] to be between $6 \times 10^{10} \mathrm{M}_{\odot}$ and $1.3 \times 10^{11} \mathrm{M}_{\odot}$, where the bulge size is $\sim 1.85 \mathrm{kpc}[78$ ]. Given the velocity dispersion in the bulge of NGC 1032, which is in the range[78] 200-225 $\mathrm{km} \mathrm{s}^{-1}$, we can also estimate the mass of the $\mathrm{MBH}$ in the nucleus using the $M-\sigma$ relation [79, 80] to be in the range $\sim(1-2) \times 10^{8} \mathrm{M}_{\odot}$. For the halo mass we use Ref. [6], which finds the virial mass of galaxies such as NGC 1032 to be $\sim 1.5 \times 10^{12} \mathrm{M}_{\odot}$ for magnitudes $-19.5<M_{B}<-20.5$ (NGC 1032 has $M_{B}=-19.8 \mathrm{mag}$ ). This mass is also generally consistent with the relation between halo mass and velocity dispersion obtained in Ref. [81] (see their Figure 3c), which gives a total galactic mass of $(2-3) \times 10^{12} \mathrm{M}_{\odot}$.

The inferred ejection velocity of the progenitor of SN 2005E from a birth place in the disk (taking the shortest distance from the observed position of SN 2005E to the plane of the galactic disk) is found to be $>1600 \mathrm{~km} \mathrm{~s}^{-1}$ for a massive $\left(\sim 25 \mathrm{M}_{\odot}\right)$, short-lived $\left(\sim 7 \times 10^{6} \mathrm{yr}\right)$ progenitor, appropriate for a SN Ib. Such an ejection velocity from the 
galactic disk would require an ejection mechanism different from that of OB runaways, where the only suggested mechanisms involve an interaction with a MBH. Such a MBH is unlikely to exist in the galactic disk. An ejection velocity of $>300 \mathrm{~km} \mathrm{~s}^{-1}$ would be required for a lower mass $\left(\sim 8 \mathrm{M}_{\odot}\right)$ and longer lived $\left(\sim 4 \times 10^{7}\right.$ yr $)$ component in a binary progenitor ejected from the disk. Such a velocity is much higher than the typical velocities of OB runaway stars[70], but it could theoretically be accessible for a runaway single star 82]. However, runaway binaries are typically ejected at lower velocities (up to $0.3-0.4$ of the maximal ejection velocities of single stars [83]), which would again suggest a different ejection mechanism than OB runaways. We conclude that the progenitor of SN $2005 \mathrm{E}$ is unlikely to be ejected from the disk by currently suggested high-velocity ejection mechanisms, and (if ejected at all) was more likely to be ejected from the centre of NGC 1032 .

For the progenitor of SN 2005E to be ejected from the galactic centre and reach its current position in the halo during its lifetime, the required ejection velocity would be at least $\sim 3400 \mathrm{~km} \mathrm{~s}^{-1}\left(\sim 1600 \mathrm{~km} \mathrm{~s}^{-1}\right)$ assuming a lifetime of $<7 \mathrm{Myr}$ for a $25 \mathrm{M}_{\odot}$ star $\left(<40 \mathrm{Myr}\right.$ for an $8 \mathrm{M}_{\odot}$ binary star). We now calculate whether such ejection velocities are likely, taking into account the conditions in NGC 1032.

We compare the velocities derived from the trajectories with the average ejection velocity of a star ejected from the galactic nucleus following the disruption of a binary by a $\mathrm{MBH}$, given by [84, 85]

$$
v_{\text {eject }}=3400 \mathrm{~km} \mathrm{~s}^{-1} \times\left(\frac{\mathrm{a}_{\text {bin }}}{0.8 \mathrm{AU}}\right)^{-1 / 2}\left(\frac{\mathrm{M}_{\text {bin }}}{50 \mathrm{M}_{\odot}}\right)^{1 / 3}\left(\frac{\mathrm{M}_{\mathrm{BH}}}{1.5 \times 10^{8} \mathrm{M}_{\odot}}\right)^{1 / 6},
$$

where $a_{\text {bin }}$ is the semimajor axis of the binary, $M_{\text {bin }}$ is the binary mass, and $M_{\mathrm{BH}}$ is the MBH mass. Massive binaries usually have components of comparable and frequently equal mass, and are known to have relatively compact orbits, with a large fraction of them $\left(f_{\text {cbin }} \approx 0.4\right)$ in close binaries $\left(a_{\text {bin }}<1 \mathrm{AU}\right.$; e.g., Refs. [86], [87], [88]). We therefore conclude that the observed position of SN 2005E is consistent with its progenitor being ejected as a massive star following the disruption of a typical very massive binary of $M_{\text {bin }}=2 \times 25=50 \mathrm{M}_{\odot}$ and $a_{\text {bin }} \lesssim 0.8 \mathrm{AU}$, or the disruption of a $M_{\text {triple }}=3 \times 8=$ $24 \mathrm{M}_{\odot}$ triple star, with an outer semimajor axis of $a_{\text {bin }} \lesssim 1.5 \mathrm{AU}$, which would eject a hypervelocity binary[89].

The velocity of an observed supernova is difficult to measure. The measured velocities of the supernova ejecta are on the order of a few thousand $\mathrm{km} \mathrm{s}^{-1}$ and the pre-explosion velocity of the progenitor is negligible, even for velocities as high as hundreds of $\mathrm{km} \mathrm{s}^{-1}$. Even for a HVS, one would require extreme velocities, directed along the line of sight, in order to identify a significant signature of the motion of the SN progenitor. The velocities we find for the ejecta of SN 2005E are not unusual, and are consistent with other SNe Ib/c.

\section{(9) The ejection rate of hypervelocity stars}


Although two massive HVS $\left(>8 \mathrm{M}_{\odot}\right.$; Refs. [73],[90]) are known in our Galaxy, it is difficult to infer the total number of Galactic massive HVSs from the very few examples known, given their serendipitous discovery nature. An estimate can be obtained if most massive HVSs in our Galaxy have been ejected through the Hills binary disruption mechanism[71, 91] (which is the likely case [92, 93, 94]). In this scenario, the binary companions of ejected HVSs should have been captured into close orbits around the MBH[95, 91]; the number of such stars should therefore reflect the number of similar HVSs in the Galaxy. Currently, a few tens of main-sequence B stars are observed in such close orbits ( $<0.04$ pc from the $\mathrm{MBH}$; e.g., Ref. [96]). Approximately half of these stars (with identified stellar types) are found to be B0-2 V main-sequence stars, most likely with masses $>8 \mathrm{M}_{\odot}$. Given the trend of massive binaries to have equal-mass components [86], one can then infer a total of $\sim 10-20$ such massive HVSs in our Galaxy. The total number of $>8 \mathrm{M}_{\odot}$ stars in the Galaxy is $\sim 10^{6}$ (e.g., assuming a Miller-Scalo initial mass function); thus, the HVS fraction of the population of massive stars is $\sim 10 / 10^{6}=10^{-5}$, and we therefore expect a similar fraction of HVS supernova progenitors. The rate of hypervelocity binary ejection is likely to be a factor of 20-100 times lower than that of single HVSs [89], making this possibility highly improbable. If the progenitor of SN 2005E were a hypervelocity single star, the probability of discovering SN 2005E in LOSS, which detected a total of $\sim 550$ core-collapse SNe from 1998 through 2008, would be low. Its discovery would then be either a chance observation of a rare event, or suggests a much higher ejection rate of extragalactic HVSs than observed in our Galaxy. However, given the low-mass ejecta observed for SN 2005E, its additional peculiarities (which cannot be explained by a hypervelocity progenitor), and the seven additional $\mathrm{SNe}$ found to have similar properties (three of them in elliptical galaxies), it is unlikely that such explosions had hypervelocity massive stellar progenitors. 


\section{(10) Comparison to SN 2002cx-like SNe}

The low luminosity and calcium-rich late-time spectra of the recently discovered SN 2008ha (see Fig. 7 of Ref. 9]) have some resemblance to the group of faint calcium-rich SNe presented here and could therefore suggest a common origin. However, SN 2008ha shares most of its observed properties with a group of peculiar SNe Ia [9, 10] (referred to as SN 2002cx-like SNe after the first example observed[4]), which are clearly distinct from the new type of explosion presented here, in almost all its observed characteristics 97] and derived properties (e.g., mass and abundances; see Table 2 for a summary of observed differences). In particular, none of the defining criteria of the SN2002cx-like group (as described by Ref. [9] and references therein) are observed for any member of the SN 2005E-like group. Specifically, none of them were observed to have a SN Ia spectral classification, very low ejecta velocities (as reflected in the line widths), early spectral resemblance to SN 1991T, slow light-curve evolution, or even the typical luminosity of 2002cx-like SNe which is $\sim 2$ mag brighter (typical absolute magnitude -17 ) than that of SN 2005E and the other members of its group $(-15 \mathrm{mag})$. Sahu et al. 68 find that SN $2005 \mathrm{hk}$, the best-observed member of the SN 2002cx-like family, requires a mass of C/O products (including $\mathrm{Si}, \mathrm{S}$, and $\mathrm{Fe}$ ) which is of order the Chandrasekhar mass. In contrast, the observation of large fractional calcium abundances (see Table 1), especially without the accompanying Si, S, and Fe (see SI, Section 4), distinguishes SN 2005E, and requires a different physical explosion mechanism - one involving helium burning. The physical distinction between the two groups is bolstered by the different stellar environments in which these events occur (see Fig. 3). 


\begin{tabular}{|c|c|c|}
\hline SNe Type & 2002cx group & 2005E group \\
\hline Spectral type & $\begin{array}{l}\text { Ia: no } \mathrm{H} \text { and } \mathrm{He} \text { lines } \\
\text { Si lines }\end{array}$ & $\begin{array}{l}\text { Ib: no } \mathrm{H} \text { and } \mathrm{Si} \text { lines } \\
\mathrm{He} \text { lines }\end{array}$ \\
\hline Absolute magnitude & -17 & -15 \\
\hline Ejecta velocity & Low & High \\
\hline $\begin{array}{c}\text { Early } \\
\text { spectra }\end{array}$ & $\begin{array}{l}\text { Resemblance to SN 1991T } \\
\text { Strong Fe-group elements }\end{array}$ & $\begin{array}{l}\text { Resemblance to regular type Ib SNe } \\
\text { No Fe-group elements }\end{array}$ \\
\hline $\begin{array}{c}\text { Late } \\
\text { spectra }\end{array}$ & $\begin{array}{l}\text { Fe-group elements } \\
\text { Intermediate elements }\end{array}$ & $\begin{array}{l}\text { Intermediate elements }(\mathrm{Ca}, \mathrm{O}) \\
\text { No Fe-group elements } \\
\text { large ratio of } \mathrm{Ca} \text { to } \mathrm{O}, \mathrm{S} \text { and } \mathrm{Ni}\end{array}$ \\
\hline Decline Rate & Slow & Fast \\
\hline Host galaxies & Late type & Early type \\
\hline
\end{tabular}

Table 2: Brief summary of the differences between the typical observed properties of SN 2002cx-like and SN 2005E-like SNe.

\section{(11) Additional calcium-rich faint type Ib/c SNe}

In addition to SN 2005E, several other objects were reported as possible members of this class of calcium-rich SNe. We have verified these reports by reinspection of the spectra, rejecting unconvincing cases, and list all verified Ca-rich events in Table 3. We also note that no radio signature has been found for any of these Ca-rich SNe (1-2 would be expected for a sample of $8 \mathrm{SNe} \mathrm{Ib}[98]$ ).

The rate of calcium-rich, faint, SNe Ib/c can be estimated since SN 2005E was discovered as part of the Lick Observatory Supernova Search (LOSS) [29]. This survey is a volume-limited search, with high sensitivity within $60 \mathrm{Mpc}$ for both SNe Ia and faint Ca-rich objects such as SN 2005E. LOSS found 2.3 calcium-rich objects (after correction for incompleteness) and 31.0 SNe Ia in this volume, from which we infer the rate of calcium-rich SNe to be $7 \% \pm 5 \%$ of the total SN Ia rate. 


\begin{tabular}{|c|c|c|c|c}
\hline SN & $\begin{array}{c}\text { Absolute B-band } \\
\text { peak magnitude }\end{array}$ & $\begin{array}{c}\text { Absolute B-band } \\
\text { discovery magnitude }\end{array}$ & Host galaxy & Host-galaxy type \\
\hline \hline $2000 \mathrm{ds}$ & $?$ & $-13.32^{*}$ & NGC 2768 & E/S0 \\
\hline $2001 \mathrm{co}$ & $-15.09^{*}$ & $-14.77^{*}$ & NGC 5559 & Sb \\
\hline $2003 \mathrm{H}$ & $?$ & $-13.43^{*}$ & NGC 2207 & Galaxy pair \\
\hline $2003 \mathrm{dg}$ & $?$ & $-15.03^{*}$ & UGC 6934 & Scd \\
\hline $2003 \mathrm{dr}$ & $-14.04^{*}$ & $-13.8^{*}$ & NGC 5714 & Sc \\
\hline $2005 \mathrm{cz}$ & $?$ & $?$ & NGC 4589 & E \\
\hline $2005 \mathrm{E}$ & -14.8 & -14.7 & NGC 1132 & S0/Sa \\
\hline $2007 \mathrm{ke}$ & $-15.45^{*}$ & $-14.7^{*}$ & NGC 1129 & E/S0 \\
\hline
\end{tabular}

Table 3: The sample of calcium-rich SNe. Discovery of these SNe is reported in Refs. 99](2000ds), [100 $(2001 \mathrm{co}), \quad$ 101] $(2003 \mathrm{H}), \quad$ 102] $(2003 \mathrm{dg}), \quad$ 103] (2003dr), 104](2005E), 105](2007ke), and [11] (2005cz).

${ }^{*} B$ magnitude unavailable; unfiltered used, corrected to $B$ using the measured colors of SN $2005 \mathrm{E}$ at peak.

A full analysis of the photometry and spectroscopy of our extended sample of faint Ca-rich SNe will be presented in a forthcoming publication.

Of the total sample of $\mathrm{SNe} \mathrm{Ib} / \mathrm{c}$ reported to reside in early-type (elliptical or $\mathrm{S} 0$ ) galaxies[13, 14, SN 2000ds and SN 2005cz are Ca-rich, faint SNe Ib. The host galaxies of all other putative SNe Ib/c in early-type hosts (SNe 2002jj, 2002hz, 2003ih, and 2006ab) were reclassified in Ref. [14] to be late-type galaxies. Thus, all confirmed $\mathrm{SNe} \mathrm{Ib/c} \mathrm{in}$ early-type hosts belong to the faint, Ca-rich type Ib class. In Fig. 3 we show the hostgalaxy distribution for the SN 2005E-like events and compare it with the distributions of other types of SNe. The hosts of SN 2005E-like events are clearly different from those of $\mathrm{SNe} \mathrm{Ib} / \mathrm{c}$ and SNe II (resulting from core collapse of a massive, short-lived star[106]) and show strong preference toward early-type host galaxies, and hence older and lower-mass progenitors. 


\section{(12) Nucleosynthetic simulations and derived abundances}

Given the unique nucleosynthetic products we observed in spectra of SN 2005E, we ran nucleosynthesis single-zone simulations [107] in order to investigate possible conditions that may lead to such signatures.

Our single-zone simulations made use of the JINA version of Reaclib (April 2009) for the rates, a 203-nucleus network, and the "explosive nucleosynthesis" procedure 107]. We investigated various initial compositions of $\mathrm{He}, \mathrm{C}$, and $\mathrm{O}$, at a density of $10^{6} \mathrm{~g} \mathrm{~cm}^{-3}$, and scanned the temperature range $(2-4.1) \times 10^{9} \mathrm{~K}$. Our main findings are illustrated in Fig. S5 and Table 4. In Fig. S4, we scale the mass fractions to fit the observed calcium abundance, and plot the observed limits on sulfur and ${ }^{56} \mathrm{Ni}$. We also present the amount of ${ }^{44} \mathrm{Ti}$ produced. A 0.6/0.4 mix of $\mathrm{He} / \mathrm{O}$ (a) works well in reproducing our data only up to $T_{9}=3.5$ where nickel becomes overabundant, and certainly fails at higher $T$, where $\mathrm{S}$ is also a problem. In the allowed phase space $\left(T_{9}<3.5\right),{ }^{44} \mathrm{Ti}$ is $0.1-0.3$ times as abundant as Ca. Increasing the amount of helium (b), permits only low temperatures $\left(T_{9}=2.2\right)$; in this case radioactive titanium and calcium are almost equally produced. Replacing oxygen by pure carbon (c) does not work for nearly equal ratios, as we either get too much $\mathrm{S}$ or too much $\mathrm{Ni}$, or both. Decreasing the amount of carbon (d) again works only for the lowest temperatures, $T_{9}=2.2$, leading to significant production of ${ }^{44} \mathrm{Ti}$.

We also note that the unique nucleosynthetic signature of SN 2005E, with evidence for $\mathrm{He}, \mathrm{O}$, and $\mathrm{Ca}$, but lacking $\mathrm{Si}, \mathrm{S}$, and $\mathrm{Fe} / \mathrm{Ni}$ differs physically (as well as in spectral appearance) from the $\mathrm{Fe} / \mathrm{Ni}$ and Si-rich ejecta of other types of $\mathrm{SNe}$ (see Table 1). Fe/Ni, $\mathrm{S}$, and $\mathrm{Si}$ are typically oxygen-burning products (common in both SNe Ia and core-collapse events), while the Ca-rich/S-poor SN 2005E is dominated by helium-burning products, a unique composition which is evidence for an underlying physical process not previously seen in other events. Such abundances are also not expected from theoretical predictions for either core collapse SNe or thermonuclear explosions of Chandrasekhar-mass WDs [108, 109 .

We conclude that helium-rich models with some $\mathrm{C} / \mathrm{O}$ contamination can recover our essential findings. A prediction is that a substantial amount of ${ }^{44} \mathrm{Ti}$ (at least 1/10 of Ca, and perhaps a comparable amount) will be synthesized. In some cases ${ }^{48} \mathrm{Cr}$ production exceeds that of ${ }^{56} \mathrm{Ni}$; its decay may therefore power the light curve[19, 110] (via ${ }^{48} \mathrm{~V}$ decay with a 16 day half-life time). A realistic model in which the burning shock traverses layers of varying temperature, density, and composition will be investigated in a forthcoming publication. 

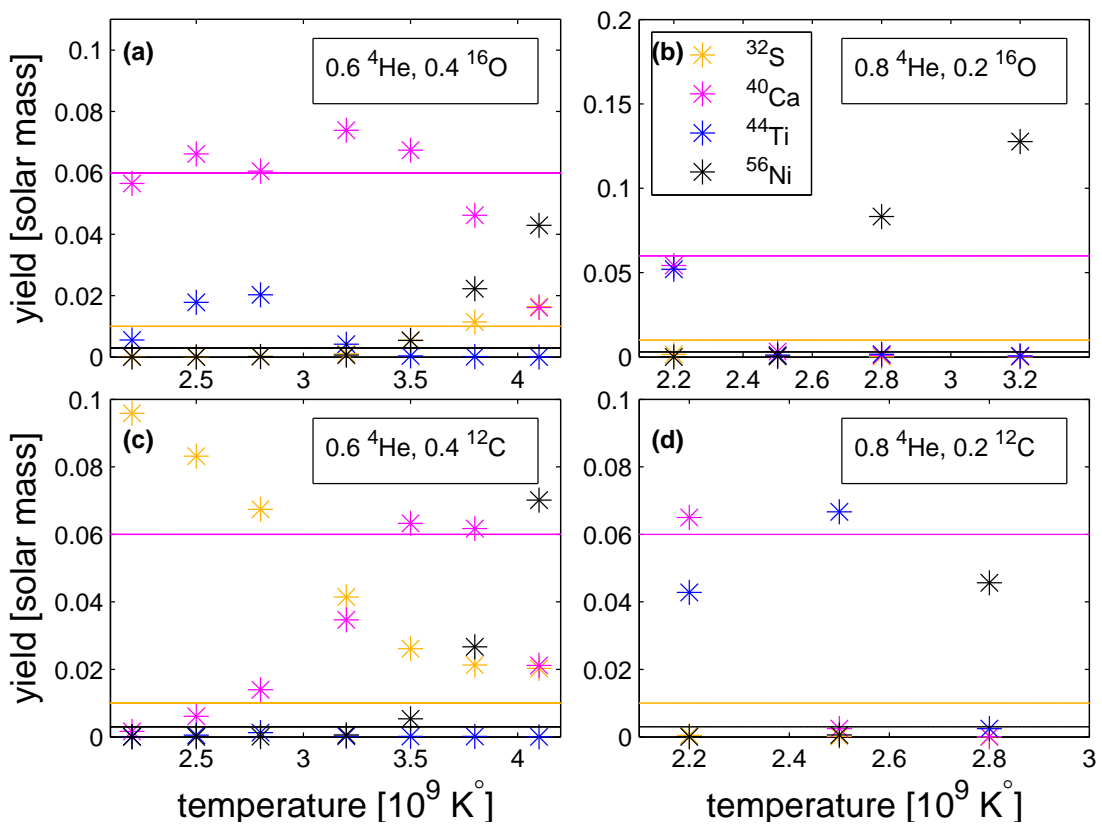

\section{Figure S5:}

Nucleosynthetic products of the burning of He-rich mixtures with carbon and oxygen (fractions marked in each panel), at various temperatures and an initial density of $10^{6} \mathrm{~g} \mathrm{~cm}^{-3}$. The fractions are scaled to best match the observed calcium mass (magenta horizontal line) and compared with the observed limit on sulfur (ocher line) and measurement of radioactive ${ }^{56} \mathrm{Ni}$ (black line). 


\begin{tabular}{|c|c|c|c|c|c|c|c|c|}
\hline \multicolumn{3}{|c|}{ Initial Conditions } & \multicolumn{6}{|c|}{ Final Values } \\
\hline$T_{9}$ & ${ }^{4} \mathrm{He}$ & ${ }^{12} \mathrm{C}$ & ${ }^{32} \mathrm{~S}$ & ${ }^{40} \mathrm{Ca}$ & ${ }^{44} \mathrm{Ti}$ & ${ }^{48} \mathrm{Cr}$ & ${ }^{52} \mathrm{Fe}$ & ${ }^{56} \mathrm{Ni}$ \\
\hline 3.4 & $8.00 \times 10^{-1}$ & $1.86 \times 10^{-1}$ & $6.18 \times 10^{-5}$ & $1.97 \times 10^{-4}$ & $2.68 \times 10^{-4}$ & $1.34 \times 10^{-4}$ & $2.82 \times 10^{-4}$ & $8.08 \times 10^{-1}$ \\
\hline 3.4 & $7.50 \times 10^{-1}$ & $2.36 \times 10^{-1}$ & $2.80 \times 10^{-4}$ & $1.30 \times 10^{-1}$ & $5.03 \times 10^{-3}$ & $9.03 \times 10^{-3}$ & $2.29 \times 10^{-1}$ & $5.82 \times 10^{-1}$ \\
\hline 3.4 & $7.00 \times 10^{-1}$ & $2.86 \times 10^{-1}$ & $2.49 \times 10^{-3}$ & $6.10 \times 10^{-1}$ & $1.60 \times 10^{-1}$ & $3.04 \times 10^{-2}$ & $1.65 \times 10^{-1}$ & $1.18 \times 10^{-1}$ \\
\hline 3.4 & $6.50 \times 10^{-1}$ & $3.36 \times 10^{-1}$ & $1.12 \times 10^{-1}$ & $5.61 \times 10^{-1}$ & $2.63 \times 10^{-3}$ & $1.84 \times 10^{-2}$ & $2.00 \times 10^{-2}$ & $2.78 \times 10^{-2}$ \\
\hline 3.4 & $6.00 \times 10^{-1}$ & $3.86 \times 10^{-1}$ & $2.09 \times 10^{-1}$ & $3.74 \times 10^{-1}$ & $1.13 \times 10^{-3}$ & $9.84 \times 10^{-3}$ & $8.01 \times 10^{-3}$ & $1.97 \times 10^{-2}$ \\
\hline 3.2 & $8.00 \times 10^{-1}$ & $1.86 \times 10^{-1}$ & $5.25 \times 10^{-5}$ & $1.74 \times 10^{-4}$ & $2.36 \times 10^{-4}$ & $1.18 \times 10^{-4}$ & $3.15 \times 10^{-4}$ & $8.29 \times 10^{-1}$ \\
\hline 3.2 & $7.50 \times 10^{-1}$ & $2.36 \times 10^{-1}$ & $1.69 \times 10^{-6}$ & $1.24 \times 10^{-1}$ & $3.94 \times 10^{-1}$ & $6.66 \times 10^{-2}$ & $4.33 \times 10^{-1}$ & $2.50 \times 10^{-1}$ \\
\hline 3.2 & $7.00 \times 10^{-1}$ & $2.86 \times 10^{-1}$ & $4.32 \times 10^{-4}$ & $6.55 \times 10^{-1}$ & $1.14 \times 10^{-1}$ & $8.00 \times 10^{-2}$ & $7.49 \times 10^{-2}$ & $1.95 \times 10^{-2}$ \\
\hline 3.2 & $6.80 \times 10^{-1}$ & $2.86 \times 10^{-1}$ & $2.73 \times 10^{-2}$ & $6.76 \times 10^{-1}$ & $2.43 \times 10^{-2}$ & $2.79 \times 10^{-2}$ & $1.37 \times 10^{-2}$ & $9.83 \times 10^{-3}$ \\
\hline 3.2 & $6.50 \times 10^{-1}$ & $3.36 \times 10^{-1}$ & $1.63 \times 10^{-1}$ & $4.18 \times 10^{-1}$ & $4.75 \times 10^{-3}$ & $1.07 \times 10^{-2}$ & $4.35 \times 10^{-3}$ & $6.86 \times 10^{-3}$ \\
\hline 3.2 & $6.00 \times 10^{-1}$ & $3.86 \times 10^{-1}$ & $2.90 \times 10^{-1}$ & $2.43 \times 10^{-1}$ & $1.56 \times 10^{-3}$ & $5.29 \times 10^{-3}$ & $1.95 \times 10^{-3}$ & $4.02 \times 10^{-3}$ \\
\hline 3.0 & $8.00 \times 10^{-1}$ & $1.86 \times 10^{-1}$ & $6.06 \times 10^{-5}$ & $2.15 \times 10^{-4}$ & $3.26 \times 10^{-4}$ & $2.38 \times 10^{-4}$ & $8.69 \times 10^{-2}$ & $7.50 \times 10^{-1}$ \\
\hline 3.0 & $7.50 \times 10^{-1}$ & $2.36 \times 10^{-1}$ & $3.85 \times 10^{-9}$ & $2.96 \times 10^{-2}$ & $1.73 \times 10^{-1}$ & $1.78 \times 10^{-1}$ & $4.53 \times 10^{-1}$ & $1.24 \times 10^{-1}$ \\
\hline 3.0 & $7.00 \times 10^{-1}$ & $2.86 \times 10^{-1}$ & $1.06 \times 10^{-3}$ & $5.72 \times 10^{-1}$ & $2.71 \times 10^{-1}$ & $4.41 \times 10^{-2}$ & $9.32 \times 10^{-3}$ & $4.16 \times 10^{-3}$ \\
\hline 3.0 & $6.50 \times 10^{-1}$ & $3.36 \times 10^{-1}$ & $2.40 \times 10^{-1}$ & $2.88 \times 10^{-1}$ & $2.91 \times 10^{-2}$ & $3.77 \times 10^{-3}$ & $7.91 \times 10^{-4}$ & $2.34 \times 10^{-3}$ \\
\hline 3.0 & $6.00 \times 10^{-1}$ & $3.86 \times 10^{-1}$ & $3.85 \times 10^{-1}$ & $1.53 \times 10^{-1}$ & $1.14 \times 10^{-1}$ & $1.51 \times 10^{-3}$ & $2.47 \times 10^{-4}$ & $1.44 \times 10^{-3}$ \\
\hline
\end{tabular}

Table 4: The abundances (by mass) of several important nuclei from post-shock burning in a heliumburning layer. A 203-nucleus network was used, with reaction rates taken from an April 2009 download of Reaclib from JINA. The initial composition was ${ }^{4} \mathrm{He},{ }^{12} \mathrm{C}$ (see table for initial values; temperature $T_{9}$ given in $10^{9} \mathrm{~K}$ ), and 0.014 of ${ }^{22} \mathrm{Ne}$ by mass. The values of ${ }^{4} \mathrm{He}$ and ${ }^{12} \mathrm{C}$ correspond to different degrees of hydrostatic helium burning prior to explosion. Low values for $\mathrm{S} / \mathrm{Ca}$ occur only for high ${ }^{4} \mathrm{He}$ abundance. For such a low S/Ca ratio, the temperature must be $T<3.2 \times 10^{9} \mathrm{~K}$ to avoid overproduction of ${ }^{56} \mathrm{Ni}$. Production of ${ }^{48} \mathrm{Cr}$ (and ${ }^{52} \mathrm{Fe}$ ) may exceed that of ${ }^{56} \mathrm{Ni}$, so that ${ }^{48} \mathrm{~V}$ decay (half-life of 16 days) may help power the light curve 19. ${ }^{44} \mathrm{Ti}$ can be copiously produced under these conditions, so that such events may provide a solution to the problem of low emission from ${ }^{44} \mathrm{Ti}$ decay in most nearby SN remnants 23,26 . The significant abundance of ${ }^{12} \mathrm{C}$ prior to the explosive burning may allow unburned (lower density) regions to explain the relatively large abundance of ${ }^{12} \mathrm{C}$ observed. To convert the abundance entries to solar mass units, multiply by the assumed mass of ejecta. 


\section{References}

[1] Filippenko, A. V. Optical spectra of supernovae. Ann. Rev. Astron. Astrophys. 35, 309-355 (1997).

[2] Mazzali, P. A. et al. A common explosion mechanism for type Ia supernovae. Science 315, 825-828 (2007).

[3] Filippenko, A. V. et al. The subluminous, spectroscopically peculiar type IA supernova 1991bg in the elliptical galaxy NGC 4374. Astron. J. 104, 1543-1556 (1992).

[4] Li, W. et al. SN 2002cx: The most peculiar known type Ia supernova. Pub. Astron. Soc. Pac. 115, 453-473 (2003).

[5] Filippenko, A. V. et al. Supernovae 2001co, 2003H, 2003dg, and 2003dr. IAU Circular 8159, 2 (2003).

[6] Prada, F. et al. Observing the dark matter density profile of isolated galaxies. Astrophys. J. 598, 260-271 (2003).

[7] Kennicutt, Jr., R. C. Star formation in galaxies along the Hubble sequence. Ann. Rev. Astron. Astrophys. 36, 189-232 (1998).

[8] Kasliwal, M. M. et al. SN 2007ax: An extremely faint type Ia supernova. Astrophys. J. Lett. 683, L29-L32 (2008).

[9] Foley, R. J. et al. SN 2008ha: An extremely low luminosity and exceptionally low energy supernova. Astron. J. 138, 376-391 (2009).

[10] Valenti, S. et al. A low-energy core-collapse supernova without a hydrogen envelope. Nature 459, 674-677 (2009).

[11] Kawabata, K. S. et al. A unique core-collapse supernova in an elliptical galaxy. ArXiv 0906.2811, (2009).

[12] Maund, J. R. \& Smartt, S. J. Hubble Space Telescope imaging of the progenitor sites of six nearby core-collapse supernovae. Mon. Not. R. Astron. Soc. 360, 288-304 (2005).

[13] van den Bergh, S., Li, W. \& Filippenko, A. V. Classifications of the host galaxies of supernovae, set III. Pub. Astron. Soc. Pac. 117, 773-782 (2005).

[14] Hakobyan, A. A. et al. Early-type galaxies with core collapse supernovae. Astron. Astrophys. 488, 523-531 (2008). 
[15] Woosley, S. E., Taam, R. E. \& Weaver, T. A. Models for type I supernova. I Detonations in white dwarfs. Astrophys. J. 301, 601-623 (1986).

[16] Woosley, S. E. \& Weaver, T. A. Sub-Chandrasekhar mass models for type Ia supernovae. Astrophys. J. 423, 371-379 (1994).

[17] Nomoto, K. \& Kondo, Y. Conditions for accretion-induced collapse of white dwarfs. Astrophys. J. Lett. 367, L19-L22 (1991).

[18] Metzger, B. D., Piro, A. L. \& Quataert, E. Nickel-rich outflows from accretion disks formed by the accretion-induced collapse of white dwarfs. Mon. Not. R. Astron. Soc. 396, 1659 (2009).

[19] Bildsten, L. et al. Faint thermonuclear supernovae from AM Canum Venaticorum binaries. Astrophys. J. Lett. 662, L95-L98 (2007).

[20] Livne, E. \& Arnett, D. Explosions of sub-Chandrasekhar mass white dwarfs in two dimensions. Astrophys. J. 452, 62-74 (1995).

[21] Iben, I. J. et al. On interacting helium star-white dwarf pairs as supernova precursors. Astrophys. J. 317, 717-723 (1987).

[22] Woosley, S. E., Arnett, W. D. \& Clayton, D. D. The explosive burning of oxygen and silicon. Astrophys. J. Supp. Ser. 26, 231-312 (1973).

[23] Timmes, F. X. et al. The production of 44Ti and 60Co in supernovae. Astrophys. J. 464, 332-341 (1996).

[24] Lai, D. K. et al. A unique star in the outer halo of the Milky Way. Astrophys. J. Lett. 697, L63-L67 (2009).

[25] de Plaa, J. et al. Constraining supernova models using the hot gas in clusters of galaxies. Astron. Astrophys. 465, 345-355 (2007).

[26] The, L.-S. et al. Are ${ }^{44}$ Ti-producing supernovae exceptional? Astron. Astrophys. 450, 1037-1050 (2006).

[27] Chan, K.-W. \& Lingenfelter, R. E. Positrons from supernovae. Astrophys. J. 405, 614-636 (1993).

[28] Knödlseder, J. et al. The all-sky distribution of $511 \mathrm{keV}$ electron-positron annihilation emission. Astron. Astrophys. 441, 513-532 (2005).

[29] Filippenko, A. V. et al. The Lick Observatory Supernova Search with the Katzman Automatic Imaging Telescope. Astronomical Society of the Pacific Conference Series 246, 121-130 (2001). 
[30] Oke, J. B. et al. The Keck low-resolution imaging spectrometer. Pub. Astron. Soc. Pac. 107, 375-385 (1995).

[31] Gal-Yam, A. et al. Preliminary Results from the Caltech Core-Collapse Project (CCCP). American Institute of Physics Conference Series 924, 297-303 (2007).

[32] Oke, J. B. \& Gunn, J. E. An efficient low resolution and moderate resolution spectrograph for the Hale telescope. Pub. Astron. Soc. Pac. 94, 586-594 (1982).

[33] Mazzali, P. A. et al. The properties of the peculiar type Ia supernova 1991bg - II. The amount of ${ }^{\wedge} 56 \mathrm{Ni}$ and the total ejecta mass determined from spectrum synthesis and energetics considerations. Mon. Not. R. Astron. Soc. 284, 151-171 (1997).

[34] Mazzali, P. A. et al. Properties of two hypernovae entering the nebular phase: SN 1997ef and SN 1997dq. Astrophys. J. 614, 858-863 (2004).

[35] Howell, D. A. et al. Gemini spectroscopy of supernovae from the supernova legacy survey: Improving high-redshift supernova selection and classification. Astrophys. J. 634, 1190-1201 (2005).

[36] Chu, Y.-H. \& Gruendl, R. A. in Massive star formation: Observations confront theory (eds Beuther, H., Linz, H. \& Henning, T.) 415-422 (ASP, 2008).

[37] Schilbach, E. \& Röser, S. On the origin of field O-type stars. Astron. Astrophys. 489, 105-114 (2008).

[38] Anderson, J. P. \& James, P. A. Constraints on core-collapse supernova progenitors from correlations with $\mathrm{H} \alpha$ emission. Mon. Not. R. Astron. Soc. 390, 1527-1538 (2008).

[39] Dyson, J. E. \& Hartquist, T. W. On the structure of intermediate- and high-velocity clouds. Mon. Not. R. Astron. Soc. 203, 1233-1238 (1983).

[40] Christodoulou, D. M., Tohline, J. E. \& Keenan, F. P. Star-forming processes far from the galactic disk: inoperative or indolent where operative. Astrophys. J. 486, 810-817 (1997).

[41] Martos, M. et al. Spiral density wave shock-induced star formation at high galactic latitudes. Astrophys. J. Lett. 526, L89-L92 (1999).

[42] Heraudeau, P. \& Simien, F. Optical and I-band surface photometry of spiral galaxies. I. The data. AAPS 118, 111-155 (1996).

[43] Kennicutt, R. C., J. \& Kent, S. M. A survey of H-alpha emission in normal galaxies. Astron. J. 88, 1094-1107 (1983). 
[44] Springob, C. M. et al. A Digital Archive of H I 21 Centimeter Line Spectra of Optically Targeted Galaxies. Astrophys. J. Supp. Ser. 160, 149-162 (2005).

[45] Welch, G. A. \& Sage, L. J. The Cool Interstellar Medium in S0 Galaxies. I. A Survey of Molecular Gas. Astrophys. J. 584, 260-277 (2003).

[46] Soderberg, A. M. The radio properties of type Ibc supernovae. American Institute of Physics Conference Series 937, 492-499 (2007).

[47] Hamuy, M. et al. Optical and infrared spectroscopy of SN 1999ee and SN 1999ex. Astron. J. 124, 417-429 (2002).

[48] Filippenko, A. V. \& Sargent, W. L. W. The unique supernova (1985f) in NGC 4618. Astron. J. 91, 691-696 (1986).

[49] Gaskell, C. M. et al. Type Ib supernovae 1983n and 1985f - Oxygen-rich late time spectra. Astrophys. J. Lett. 306, L77-L80 (1986).

[50] Nadyozhin, D. K. Explosion energies, nickel masses and distances of type II plateau supernovae. Mon. Not. R. Astron. Soc. 346, 97-104 (2003).

[51] Mazzali, P. A., Iwamoto, K. \& Nomoto, K. A spectroscopic analysis of the energetic type Ic Hypernova SN 1997EF. Astrophys. J. 545, 407-419 (2000).

[52] Tominaga, N. et al. The unique type Ib supernova 2005bf: A WN star explosion model for peculiar light curves and spectra. Astrophys. J. Lett. 633, L97-L100 (2005).

[53] Zampieri, L. Physical properties of type II supernovae and their progenitors. Astronomical Society of the Pacific Conference Series 342, 358-365 (2005).

[54] Li, L.-X. Correlation between the peak spectral energy of gamma-ray bursts and the peak luminosity of the underlying supernovae: implication for the nature of the gamma-ray burst-supernova connection. Mon. Not. R. Astron. Soc. 372, 1357-1365 (2006).

[55] Sauer, D. N. et al. The properties of the 'standard' Type Ic supernova 1994I from spectral models. Mon. Not. R. Astron. Soc. 369, 1939-1948 (2006).

[56] Mazzali, P. A. et al. The aspherical properties of the energetic type Ic SN 2002ap as inferred from its nebular spectra. Astrophys. J. 670, 592-599 (2007).

[57] Utrobin, V. P., Chugai, N. N. \& Pastorello, A. Ejecta and progenitor of the lowluminosity type IIP supernova 2003Z. Astron. Astrophys. 475, 973-979 (2007). 
[58] Soderberg, A. M. et al. An HST study of the supernovae accompanying GRB 040924 and GRB 041006. Astrophys. J. 636, 391-399 (2006).

[59] Pastorello, A. et al. Low-luminosity Type II supernovae: spectroscopic and photometric evolution. Mon. Not. R. Astron. Soc. 347, 74-94 (2004).

[60] Arnett, W. D. Type I supernovae. I - Analytic solutions for the early part of the light curve. Astrophys. J. 253, 785-797 (1982).

[61] Stehle, M. et al. Abundance stratification in Type Ia supernovae - I. The case of SN 2002bo. Mon. Not. R. Astron. Soc. 360, 1231-1243 (2005).

[62] Modjaz, M. et al. From shock breakout to peak and beyond: Extensive panchromatic observations of the aspherical type Ib supernova 2008D associated with Swift X-ray transient 080109. Astrophys. J. 702, 226 (2009).

[63] Cenko, S. B. et al. The Automated Palomar 60 Inch Telescope. Pub. Astron. Soc. Pac. 118, 1396-1406 (2006).

[64] NED Nasa/ipac extragalactic database http://nedwww.ipac.caltech.edu/.

[65] Tanaka, M., Maeda, K., Mazzali, P. A. \& Nomoto, K. Multidimensional Simulations for Early-Phase Spectra of Aspherical Hypernovae: SN 1998bw and Off-Axis Hypernovae. Astrophys. J. Lett. 668, L19-L22 (2007).

[66] Mazzali, P. A. et al. Keck and European Southern Observatory Very Large Telescope View of the Symmetry of the Ejecta of the XRF/SN 2006aj. Astrophys. J. 661, 892-898 (2007).

[67] Sauer, D. N. \& Mazzali, P. A. Interpretation of observed type Ia supernova spectra with radiative transfer models. New Astron. Rev. 52, 370-372 (2008).

[68] Sahu, D. K. et al. The evolution of the peculiar type Ia supernova SN 2005hk over 400 days. Astrophys. J. 680, 580-592 (2008).

[69] Blaauw, A. On the origin of the O- and B-type stars with high velocities (the "runaway" stars), and some related problems. Bulletin of the Astronomical Institutes of the Netherlands 15, 265-290 (1961).

[70] Hoogerwerf, R., de Bruijne, J. H. J. \& de Zeeuw, P. T. On the origin of the $\mathrm{O}$ and B-type stars with high velocities. II. Runaway stars and pulsars ejected from the nearby young stellar groups. Astron. Astrophys. 365, 49-77 (2001).

[71] Hills, J. G. Hyper-velocity and tidal stars from binaries disrupted by a massive Galactic black hole. Nature 331, 687-689 (1988). 
[72] Brown, W. R. et al. Discovery of an unbound hypervelocity star in the Milky Way halo. Astrophys. J. Lett. 622, L33-L36 (2005).

[73] Edelmann, H. et al. HE 0437-5439: An unbound hypervelocity main-sequence BType star. Astrophys. J. Lett. 634, L181-L184 (2005).

[74] Hirsch, H. A. et al. US 708 - an unbound hyper-velocity subluminous O star. Astron. Astrophys. 444, L61-L64 (2005).

[75] Eisenhauer, F. et al. SINFONI in the Galactic center: Young stars and infrared flares in the central light-month. Astrophys. J. 628, 246-259 (2005).

[76] Ghez, A. M. et al. Stellar orbits around the Galactic center black hole. Astrophys. J. 620, 744-757 (2005).

[77] Miyamoto, M. \& Nagai, R. Three-dimensional models for the distribution of mass in galaxies. Publ. of the Astronomical Society of Japan 27, 533-543 (1975).

[78] Gorgas, J., Jablonka, P. \& Goudfrooij, P. Stellar population gradients in bulges along the Hubble sequence. I. The data. Astron. Astrophys. 474, 1081-1092 (2007).

[79] Ferrarese, L. \& Merritt, D. A fundamental relation between supermassive black holes and their host galaxies. Astrophys. J. Lett. 539, L9-L12 (2000).

[80] Gebhardt, K. et al. A relationship between nuclear black hole mass and galaxy velocity dispersion. Astrophys. J. Lett. 539, L13-L16 (2000).

[81] Shankar, F. et al. New relationships between galaxy properties and host halo mass, and the role of feedbacks in galaxy formation. Astrophys. J. 643, 14-25 (2006).

[82] Leonard, P. J. T. The maximum possible velocity of dynamically ejected runaway stars. Astron. J. 101, 562-571 (1991).

[83] Leonard, P. J. T. \& Duncan, M. J. Runaway stars from young star clusters containing initial binaries. II - A mass spectrum and a binary energy spectrum. Astron. J. 99, 608-616 (1990).

[84] Hills, J. G. Computer simulations of encounters between massive black holes and binaries. Astron. J. 102, 704-715 (1991).

[85] Bromley, B. C. et al. Hypervelocity stars: Predicting the spectrum of ejection velocities. Astrophys. J. 653, 1194-1202 (2006).

[86] Abt, H. A. Normal and abnormal binary frequencies. Ann. Rev. Astron. Astrophys. 21, 343-372 (1983). 
[87] Morrell, N. \& Levato, H. Spectroscopic binaries in the Orion OB1 association. Astrophys. J. Supp. Ser. 75, 965-985 (1991).

[88] Kobulnicky, H. A. \& Fryer, C. L. A new look at the binary characteristics of massive stars. Astrophys. J. 670, 747-765 (2007).

[89] Perets, H. B. Runaway and hypervelocity stars in the Galactic halo: Binary rejuvenation and triple disruption. Astrophys. J. 698, 1330-1340 (2009).

[90] Heber, U. et al. The B-type giant HD 271791 in the Galactic halo. Linking run-away stars to hyper-velocity stars. Astron. Astrophys. 483, L21-L24 (2008).

[91] Yu, Q. \& Tremaine, S. Ejection of hypervelocity stars by the (binary) black hole in the Galactic center. Astrophys. J. 599, 1129-1138 (2003).

[92] Perets, H. B., Hopman, C. \& Alexander, T. Massive perturber-driven interactions between stars and a massive black hole. Astrophys. J. 656, 709-720 (2007).

[93] Perets, H. B. Dynamical and evolutionary constraints on the nature and origin of hypervelocity stars. Astrophys. J. 690, 795-801 (2009).

[94] Perets, H. B. et al. Dynamical evolution of the young stars in the Galactic center: N-body simulations of the S-Stars. Astrophys. J. 702, 884-889 (2009).

[95] Gould, A. \& Quillen, A. C. Sagittarius A* companion S0-2: A probe of very high mass star formation. Astrophys. J. 592, 935-940 (2003).

[96] Gillessen, S. et al. Monitoring stellar orbits around the massive black hole in the Galactic center. Astrophys. J. 692, 1075-1109 (2009).

[97] Jha, S. et al. Late-time spectroscopy of SN 2002cx: The prototype of a new subclass of type Ia supernovae. Astron. J. 132, 189-196 (2006).

[98] Soderberg, A. M. et al. Late-time radio observations of 68 type Ibc supernovae: Strong constraints on off-Axis gamma-ray bursts. Astrophys. J. 638, 930-937 (2006).

[99] Puckett, T. \& Dowdle, G. Supernova 2000ds in NGC 2768. IAU circulars 7507, 2 (2000).

[100] Aazami, A. B. \& Li, W. D. Supernova 2001co in NGC 5559. IAU circulars 7643, 2 (2001).

[101] Graham, J. et al. Supernovae 2003E, 2003F, 2003G, 2003H. IAU circulars 8045, 1 (2003). 
[102] Pugh, H. \& Li, W. Supernova 2003dg in UGC 6934. IAU circulars 8113, 2 (2003).

[103] Puckett, T. et al. Supernovae 2003dm, 2003dn, 2003do, 2003dp, 2003dq, 2003dr. IAU circulars $\mathbf{8 1 1 7 ,} 1$ (2003).

[104] Graham, J. et al. Supernovae 2005E, 2005F, 2005G, 2005H, 2005I, 2005J. IAU circulars 8467, 1 (2005).

[105] Chu, J. \& Li, W. Supernova 2007ke in NGC 1129. Central Bureau Electronic Telegrams 1084, 1 (2007).

[106] Smartt, S. J. Progenitors of Core-Collapse Supernovae. Ann. Rev. Astron. Astrophys. 47, 63-106 (2009).

[107] Arnett, D. Supernovae and nucleosynthesis. an investigation of the history of matter, from the Big Bang to the present. Princeton series in astrophysics, Princeton, NJ: Princeton University Press (1996).

[108] Nomoto, K. et al. Nucleosynthesis in type Ia supernovae. Nuclear Physics A 621 , 467-476 (1997).

[109] Nomoto, K. et al. Nucleosynthesis in type II supernovae. Nuclear Physics A 616 , 79-90 (1997).

[110] Shen, K. J. \& Bildsten, L. Unstable helium shell burning on accreting white dwarfs. Astrophys. J. 699, 1365 (2009). 

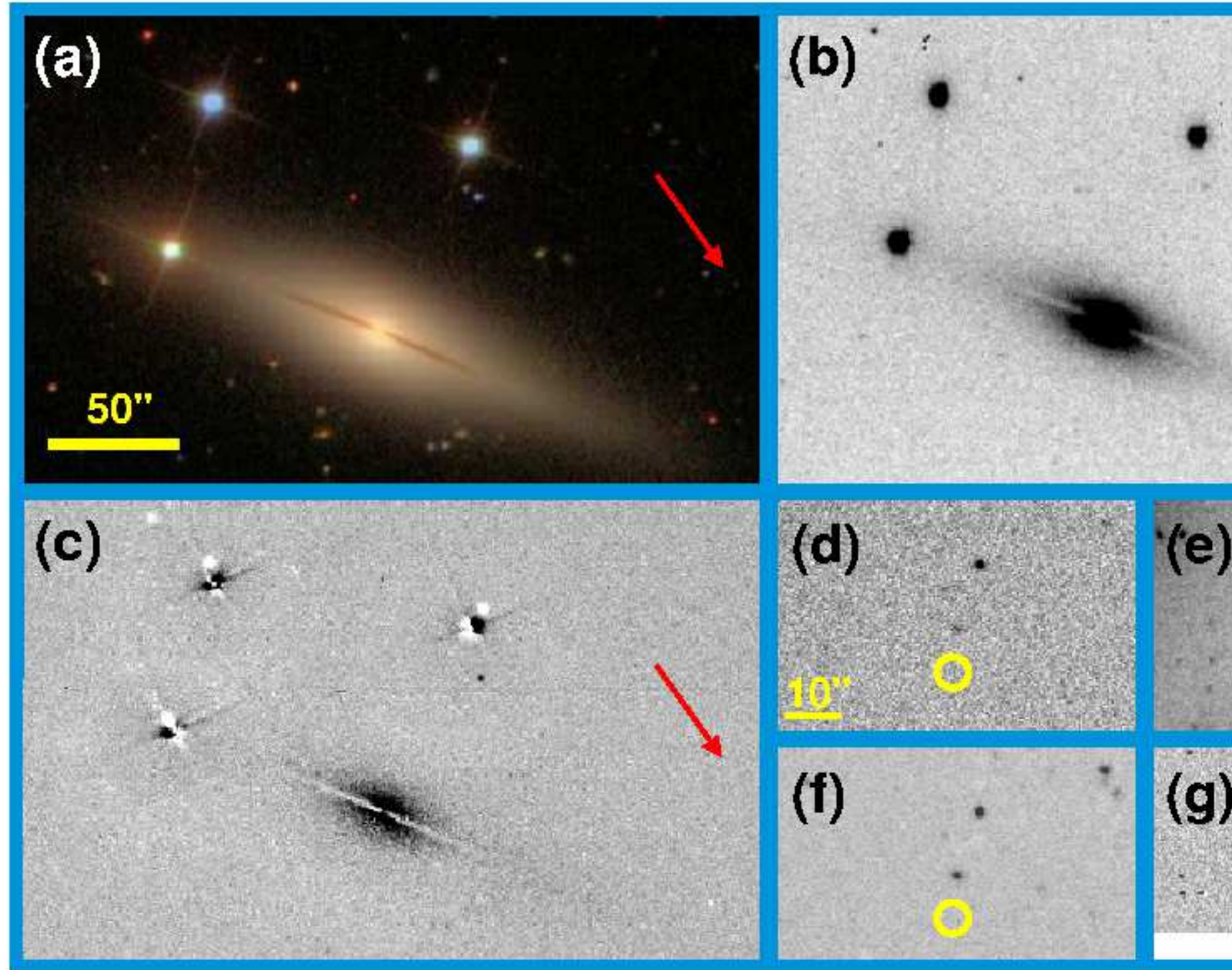

(e)

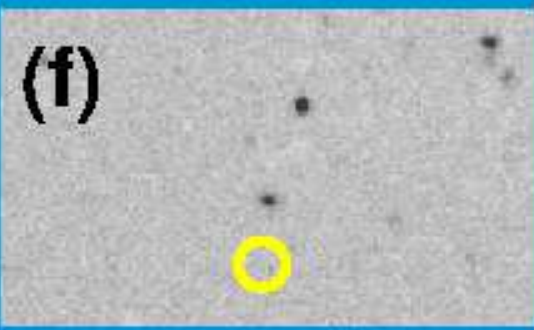

(g) 


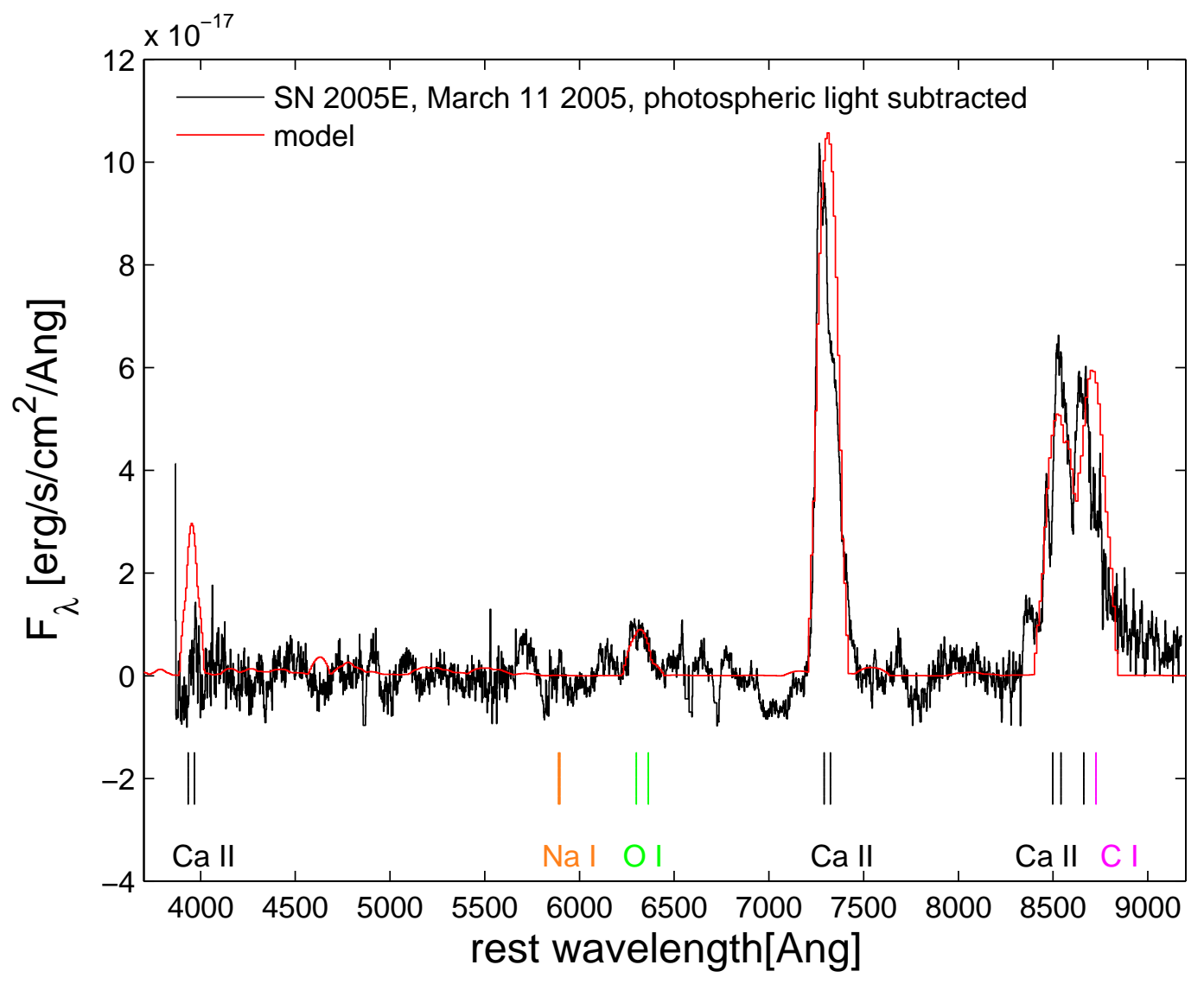




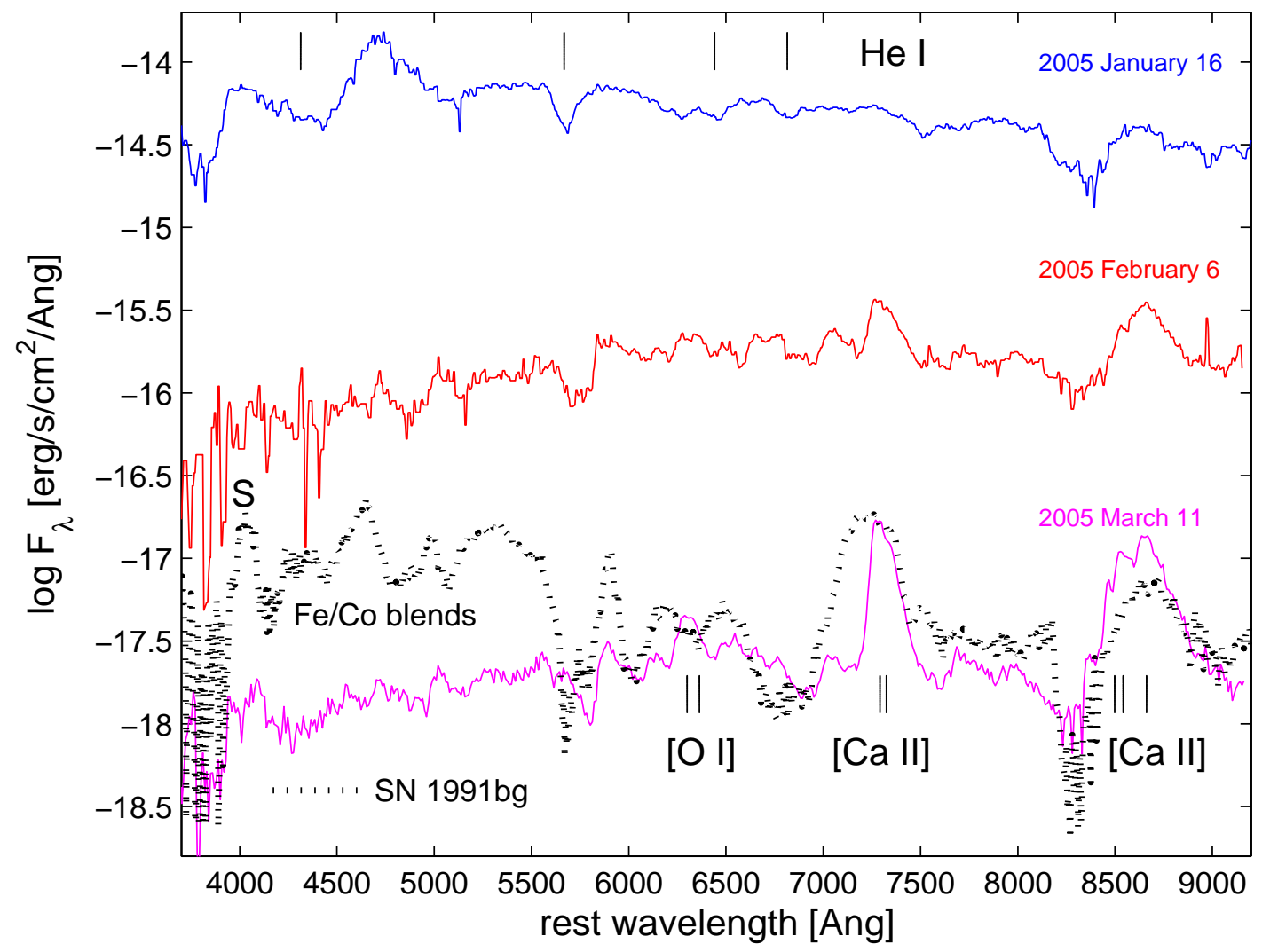

